University of New Hampshire

University of New Hampshire Scholars' Repository

Coronal Mass Ejection Research Group

Institute for the Study of Earth, Oceans, and

Space (EOS)

$10-23-2012$

\title{
The Heliospheric Plasma Sheet Observed in situ by Three Spacecraft over Four Solar Rotations
}

\author{
K. D. C. Simunac \\ University of New Hampshire \\ Antoinette B. Galvin \\ University of New Hampshire, toni.galvin@unh.edu \\ Charlie J. Farrugia \\ University of New Hampshire, Charlie.Farrugia@unh.edu \\ L. M. Kistler \\ University of New Hampshire \\ Harald Kucharek \\ University of New Hampshire, Harald.Kucharek@unh.edu
}

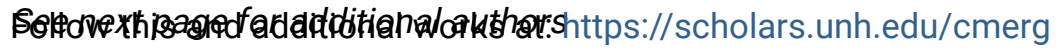
Comments

This is an article published by Springer in Solar Physics in 2012, available online: https://dx.doi.org/10.1007/

s11207-012-0156-9

\section{Recommended Citation}

Simunac, K. D. C.; Galvin, A. B.; Farrugia, C. J.; Kistler, L. M.; Kucharek, H.; Lavraud, B.; Liu, Y. C. -M.; Luhmann, J. G.; Ogilvie, K. W.; Opitz, A.; Popecki, M. A.; Sauvaud, J. -A.; Wang, S. (2012). The Heliospheric Plasma Sheet Observed in situ by Three Spacecraft over Four Solar Rotations, SOLAR PHYSICS. Vol. 281, No. 1, 423-447. DOI: 10.1007/s11207-012-0156-9

This Article is brought to you for free and open access by the Institute for the Study of Earth, Oceans, and Space (EOS) at University of New Hampshire Scholars' Repository. It has been accepted for inclusion in Coronal Mass Ejection Research Group by an authorized administrator of University of New Hampshire Scholars' Repository. For more information, please contact Scholarly.Communication@unh.edu. 


\section{Authors}

K. D. C. Simunac, Antoinette B. Galvin, Charlie J. Farrugia, L. M. Kistler, Harald Kucharek, B. Lavraud, Y. C. M. Liu, J. G. Luhmann, K. W. Ogilivie, A. Opitz, M. A. Popecki, J. A. Sauvaud, and S. Wang 


\title{
The Heliospheric Plasma Sheet Observed in situ by Three Spacecraft over Four Solar Rotations
}

\author{
K.D.C. Simunac · A.B. Galvin • C.J. Farrugia - L.M. Kistler · H. Kucharek • \\ B. Lavraud · Y.C.-M. Liu · J.G. Luhmann · K.W. Ogilvie • A. Opitz • M.A. Popecki • \\ J.-A. Sauvaud · S. Wang
}

Received: 4 November 2011 / Accepted: 25 September 2012 / Published online: 23 October 2012

(C) The Author(s) 2012. This article is published with open access at Springerlink.com

\begin{abstract}
In this paper we present in situ observations of the heliospheric plasma sheet (HPS) from STEREO-A, Wind, and STEREO-B over four solar rotations in the declining phase of Solar Cycle 23, covering late March through late June 2007. During this time period the three spacecraft were located in the ecliptic plane, and were gradually separating in heliographic longitude from about 3 degrees to 14 degrees. Crossings of the HPS were identified using the following criteria: reversal of the interplanetary magnetic field sector, enhanced proton density, and local minima in both the proton specific entropy argument and in the alpha particle-to-proton number density ratio $\left(N_{\mathrm{a}} / N_{\mathrm{p}}\right)$. Two interplanetary coronal mass ejections (ICMEs) were observed during the third solar rotation of our study period, which disrupted the HPS from its quasi-stationary state. We find differences in the in situ proton parameters at the HPS between the three spacecraft despite temporal separations of less than one day. We attribute these differences to both small separations in heliographic latitude and radial evolution of the solar wind leading to the development of compression
\end{abstract}

The Sun 360

Guest Editors: Bernhard Fleck, Bernd Heber, and Angelos Vourlidas

K.D.C. Simunac $(\bowtie)$ - A.B. Galvin · C.J. Farrugia · L.M. Kistler · H. Kucharek · Y.C.-M. Liu •

M.A. Popecki $\cdot$ S. Wang

University of New Hampshire, Durham, NH, USA

e-mail: K.Simunac@unh.edu

J.G. Luhmann

University of California, Berkeley, CA, USA

K.W. Ogilvie

NASA Goddard Space Flight Center, Greenbelt, MD, USA

B. Lavraud · A. Opitz · J.-A. Sauvaud

IRAP (CNRS-UPS), University of Toulouse, Toulouse, France

Y.C.-M. Liu

State Key Laboratory of Space Weather, Center for Space Science and Applied Research,

Chinese Academy of Sciences, Beijing, China 
regions associated with stream interaction regions (SIRs). We also observed a modest enhancement in the density of iron ions at the HPS.

\section{Introduction}

Since the 1960s, in situ spacecraft measurements of the interplanetary magnetic field (IMF) have shown the direction of the IMF in the ecliptic plane alternates between "towards" and "away" sectors as a quasi-stationary structure that co-rotates with the Sun (Wilcox and Ness, 1965). Threading through interplanetary space between the regions of opposite polarity is the heliospheric current sheet (HCS), which was found to correlate with the streamer belt at the Sun (Gosling et al., 1981; Borrini et al., 1981). More recently, Winterhalter et al. (1994), Crooker et al. (2004), and Suess et al. (2009) found that the HCS is embedded in, or adjacent to, a slow solar wind with enhanced proton density: the heliospheric plasma sheet (HPS).

Enhanced proton density and a change in magnetic sector are not the only in situ identifiers of the HPS. Burlaga, Mish, and Whang (1990), and Siscoe and Intriligator (1993) noted a drop in entropy correlated with the HPS. Winterhalter et al. (1994) found enhanced plasma $\beta$ to be another criterion for HPS identification. A local minimum in alpha particleto-proton number density ratio $\left(N_{\mathrm{a}} / N_{\mathrm{p}}\right)$ was found in the studies of Borrini et al. (1981), Geiss, Gloeckler, and von Steiger (1995), Bavassano, Woo, and Bruno (1997), and Suess et al. (2009). Suess et al. (2009) and Liu et al. (2010a) found a local depletion in the oxygento-proton number density ratio at the HPS. Suess et al. (2009) also found a possible increase in the average iron charge state at the HPS, noting that it could be a remnant of interplanetary coronal mass ejection (ICME) material.

In this paper we present in situ observations of a recurring HPS crossing over four solar rotations: Carrington rotations (CRs) 2054 through 2057. The data presented here were acquired between March and June 2007 by three spacecraft: the STEREO observatories (ST-A and ST-B; see Kaiser et al., 2008), and Wind (see Acuña et al., 1995). Throughout the observation period the three spacecraft were at similar heliographic latitudes (essentially in the ecliptic plane), while their heliographic longitudinal separation increased from three degrees to 14 degrees. Figure 1 shows the modeled neutral line (solid black curve; courtesy of the GONG consortium) for the four CRs, overlaid on synoptic maps of the corona at a wavelength of $195 \AA$ from ST-A/SECCHI/EUVI (Howard et al., 2008). The projected trajectories of the three spacecraft are shown as nearly horizontal lines in red (ST-A), blue (ST-B), and green (Wind). From top to bottom the panels show CRs 2054, 2055, 2056, and 2057. The sector boundary region of interest is indicated with a light blue oval in each panel. Time runs from right to left, so the spacecraft will be crossing from the "away" polarity of the southern hemisphere to the "towards" polarity of the northern hemisphere in the indicated region.

\section{Data}

The observations presented here are from ST-A, Wind, and ST-B. Wind plasma and electron pitch angle data are from the Solar Wind Experiment (SWE, Ogilvie et al., 1995). Wind magnetic field data are from the Magnetic Field Investigation (MFI, Lepping et al., 1995). STEREO magnetic field data are from the Magnetic Field Experiment (MAG, Acuña et al., 2008). Electron pitch angle distributions (PADs) are from the Solar Wind Electron Analyzer 
Figure 1 Modeled HCS (solid black curve, courtesy of the GONG consortium) overlaid on synoptic maps of the corona at $195 \AA$ from ST-A/SECCHI. The projected spacecraft trajectories are the nearly horizontal lines. Red is ST-A, blue is ST-B, and green is Wind. CR No. 2054 through 2057 are shown from top to bottom, with the sector boundary crossing of interest indicated by the light blue ovals.
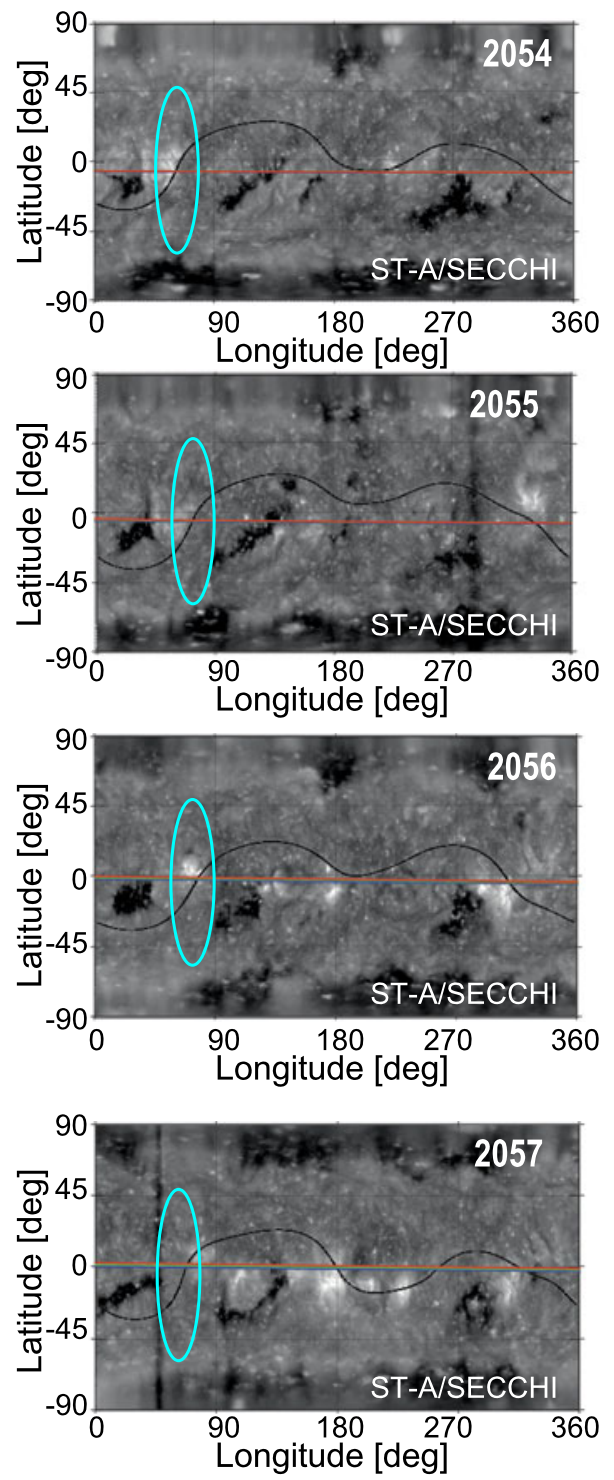

(SWEA, Sauvaud et al., 2008). MAG and SWEA are both part of the In-Situ Measurements of Particles and CME Transients (IMPACT) suite (Luhmann et al., 2008). Plasma data are from the Plasma and Suprathermal Ion Composition (PLASTIC) experiment (Galvin et al., 2008). The process by which the PLASTIC solar wind plasma data were obtained is described below.

The PLASTIC investigation is novel in that each flight unit functions like three separate instruments: a solar wind proton/alpha monitor, a solar wind heavy ion composition experiment, and a plasma and suprathermal composition sensor away from the Sun-spacecraft line. Multi-functionality gives rise to a need for multiple geometric factors and fields-of- 
view within the same instrument. The first major subdivision within the instrument is based upon the in-ecliptic (azimuth) field-of-view. The region that covers the Sun-spacecraft line \pm 22.5 degrees is the Solar Wind Sector (SWS). The remaining portion is called the Wide Angle Partition (WAP). All the data presented here are from the SWS. The SWS can be further subdivided into two sections based upon entrance aperture: the larger geometric factor "Main" channel intended for heavy ions, and the smaller geometric factor "S" channel for more abundant solar wind species such as protons. The PLASTIC electro-static analyzer (ESA) steps through logarithmically spaced energy-per-charge $(E / Q)$ steps from about $80 \mathrm{keV} \mathrm{e}^{-1}$, down to about $0.2 \mathrm{keV} \mathrm{e}^{-1}$. At the start of each ESA sweep the small channel is gated closed with an electric field, and the Main channel is open. Upon reaching a set (but command-able) count threshold, the Main channel is closed and the S channel is opened. The larger geometric factor in the Main channel allows for sufficient counting statistics of heavy solar wind ions, and the switch to the smaller geometric factor prevents the electronics and solid-state detectors from being inundated with the high-flux solar wind protons. In-flight data show evidence that when the $\mathrm{S}$ channel entrance is in use a higher than expected fluence of low $E / Q$ ions is obtained (based on pre-launch testing and calibration). The distribution of counts appears to be bifurcated in azimuth angle when the $\mathrm{S}$ channel is engaged. This behavior has been linked to insufficient fringe-field control in the upper section of the entrance system, which results in only partial suppression of ions through the main channel gate. Proton data have been recalibrated after an extensive in-flight characterization of the S channel behavior. (See Opitz (2007) and Simunac (2009).) Iron data were acquired in the main channel, where this is not an issue and pre-launch calibration is applicable.

The data analyzed in this study were collected from late March to late June 2007, part of the declining phase of Solar Cycle 23. Figures 2a, 2b, and 2c show data from ST-A, ST-B, and Wind (respectively) covering 31 March through 1 April 2007. This time period corresponds to the first HPS crossing (CR 2054) of the series. From top to bottom the panels are: electron pitch angle distribution (PAD) $[\mathrm{deg}]$, proton density $\left[\mathrm{cm}^{-3}\right]$ and proton specific entropy argument (defined as $\left.\mathrm{T}^{-1 / 2}\right)$ scaled by $10^{-4}\left[\mathrm{~K} \mathrm{~cm}^{-3 / 2}\right]$, the radial $\left(B_{\mathrm{r}}\right.$ or $\left.B_{x}\right)$ and tangential $\left(B_{\mathrm{t}}\right.$ or $\left.B_{y}\right)$ magnetic field components [nT], alpha to proton number density ratio $\left(N_{\mathrm{a}} / N_{\mathrm{p}}\right.$; a unitless quantity) and solar wind proton speed $\left[\mathrm{km} \mathrm{s}^{-1}\right]$, and lastly the iron ion density. The iron density at ST-A is scaled by a factor of $10^{6}\left[\mathrm{~cm}^{-3}\right]$. The iron density at ST-B is shown in arbitrary units. The iron density is not available from the Wind spacecraft, so the iron density observed by the SWICS instrument (Gloeckler et al., 1998) aboard ACE (Stone et al., 1998) is shown in the bottom panel of Figure 2c. (ACE and Wind were both near L1.) The regions in Figures 2a, 2b, and 2c shaded in light magenta correspond to the heliospheric plasma sheet (HPS). The heliospheric current sheet (HCS) is shown as a vertical dashed line. The process used to identify the HPS for this study is described in Section 3, using the first HPS crossing at ST-A as an example.

\section{Plasma Sheet Identification}

In order to confirm that we identified a true sector boundary (rather than a local field reversal), we examined both the magnetic field data and the electron PAD ( $c f$. Crooker et al., 1996). ST-A crossed the HCS on 31 March around 22:15 UT, when the electron flux distribution changed from predominantly 0 degrees to predominantly 180 degrees. This switch in the PAD from the "away" to the "towards" polarity shows that we have identified a true sector boundary. In this case the sector boundary agrees with the 


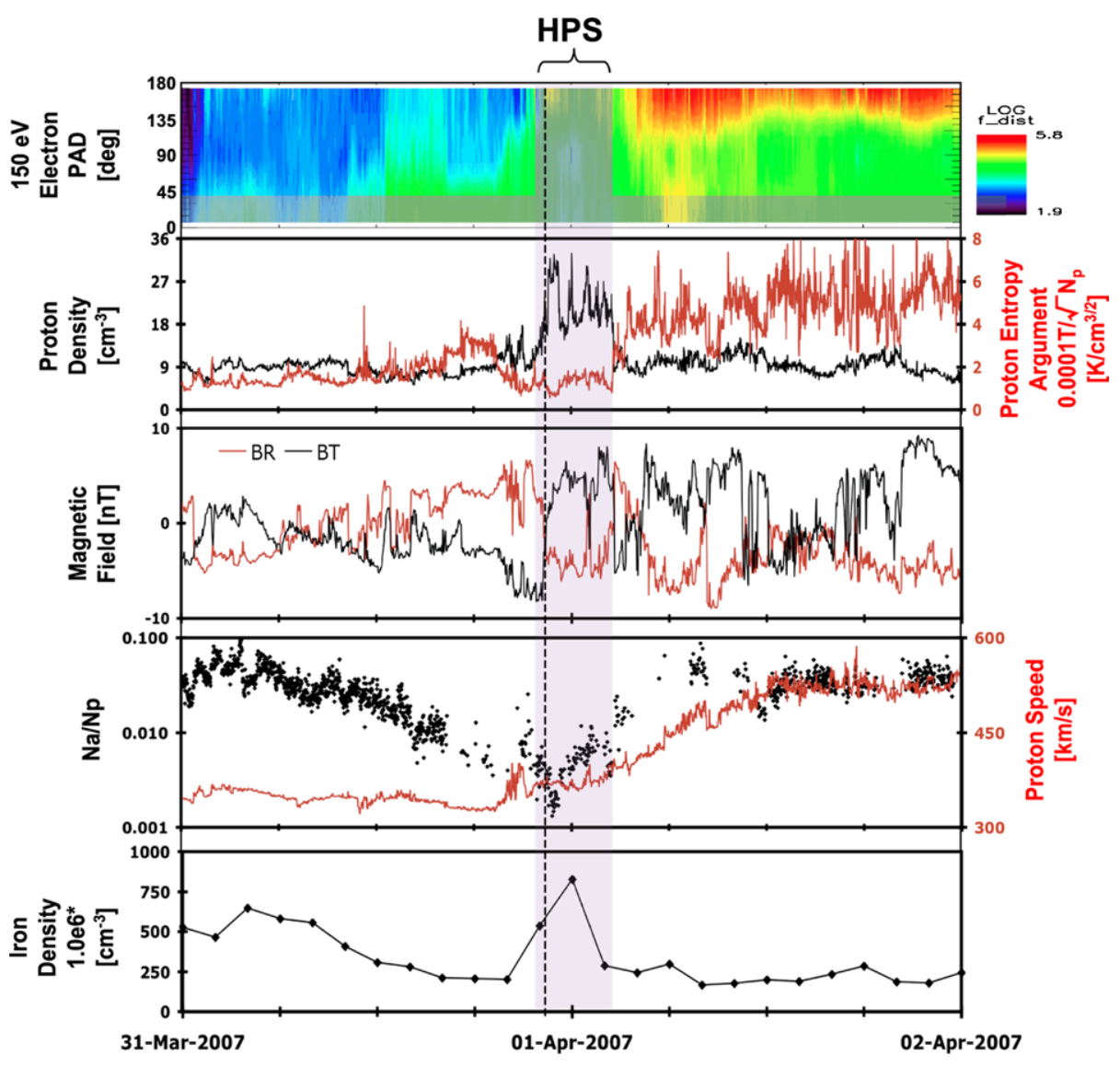

(a)

Figure 2 (a) ST-A in situ data from the first studied HPS crossing. From top to bottom the panels are: $150 \mathrm{eV}$ electron PADs, proton density (black) and proton entropy argument (red), magnetic field components BR (red) and BT (black), $N_{\mathrm{a}} / N_{\mathrm{p}}$ (black) and solar wind proton speed (red), and iron ion density. The magenta shaded region near the center is the HPS. The dashed vertical line is the HCS. (b) ST-B in situ data from the first studied HPS crossing. From top to bottom the panels are: $150 \mathrm{eV}$ electron PADs, proton density (black) and proton entropy argument (blue), magnetic field components BR (blue) and BT (black), and $N_{\mathrm{a}} / N_{\mathrm{p}}$ (black) with solar wind proton speed (blue), and iron ion density. The magenta shaded region near the center is the HPS. The dashed vertical line is the HCS. (c) Wind in situ data from the first studied HPS crossing. From top to bottom the panels are: $193 \mathrm{eV}$ electron PADs, proton density (black) and proton entropy argument (green), magnetic field components $B_{x}$ (green) and $B_{y}$ (black), and $N_{\mathrm{a}} / N_{\mathrm{p}}$ (black) with solar wind proton speed (green). The bottom panel shows the iron ion density from ACE/SWICS. The magenta shaded region near the center is the HPS. The dashed vertical line is the HCS.

location of the HCS, which is shown with a vertical dashed line in Figure 2. At the HCS the radial and tangential magnetic fields both undergo and sustain a change in polarity. To identify the associated HPS we look for increased proton density (Winterhalter et al., 1994), accompanied by low proton entropy (Burlaga, Mish, and Whang, 1990; Siscoe and Intriligator, 1993) and a drop in $N_{\mathrm{a}} / N_{\mathrm{p}}$ (Borrini et al., 1981; Geiss, Gloeckler, and von Steiger, 1995; Suess et al., 2009).

During the period in which the proton density is enhanced in Figure 2a, the proton entropy argument reaches a local minimum, and the alpha to proton ratio is minimized. We 


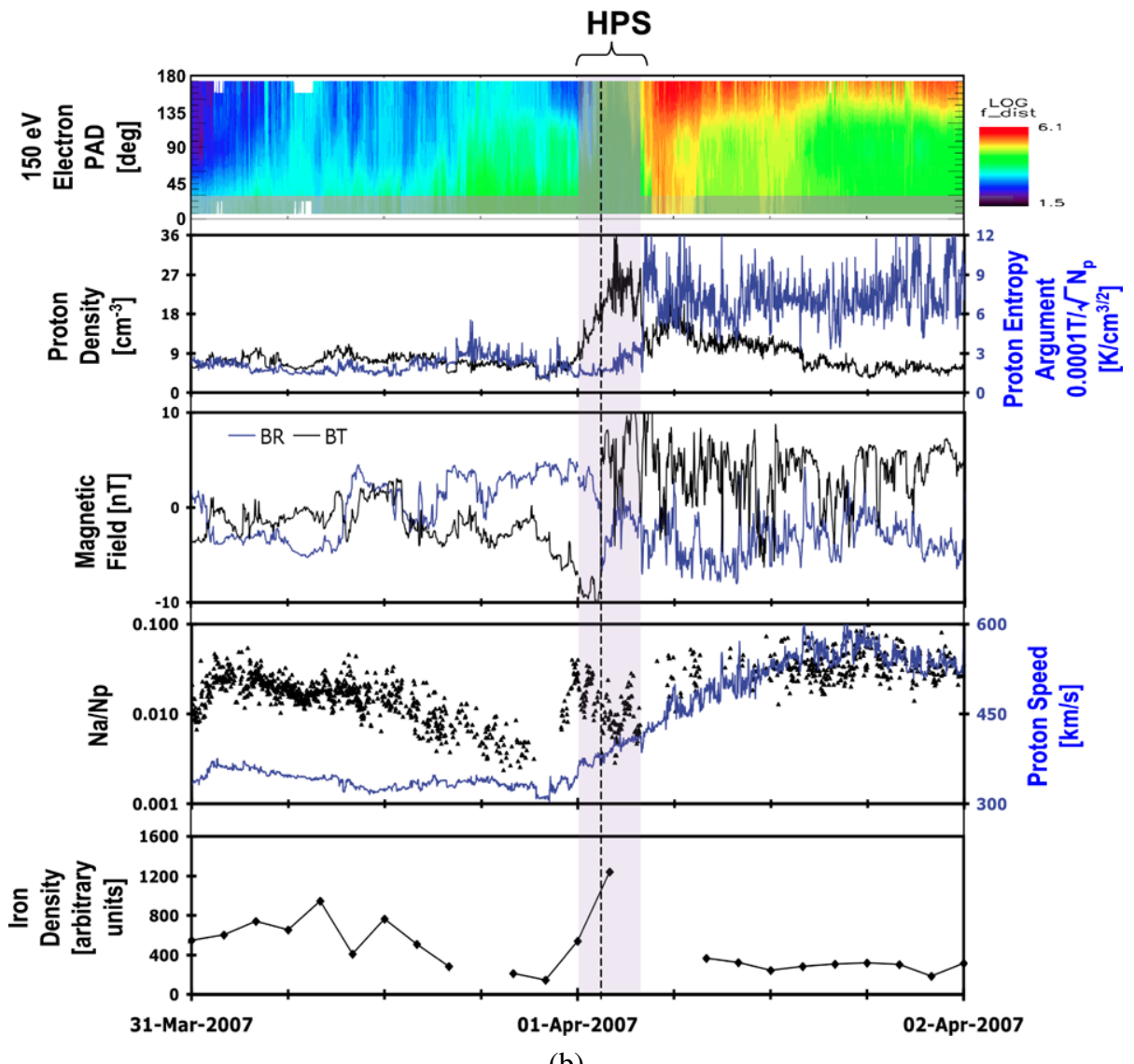

Figure 2 (Continued.)

interpret this region as the HPS for event identification. This process was repeated for the events in this study listed in Table 1. The radial (i.e. along the Sun-spacecraft line) extent or "width" of the HPS at each spacecraft provided in Table 1 was estimated by multiplying the temporal duration by the average solar wind speed observed during that period. (The error bars are based upon the uncertainty in the measured solar wind speed.) The HPS boundaries were difficult to identify with confidence during CR 2056 because of the intrusion of two transients, so they are not included in Table 1. This will be discussed further in Sections 4 and 5.

\section{Observations}

\subsection{CROSSING 1, CR 2054}

The first of the studied HPS crossings (see Figures 2a, 2b, and 2c) took place from 31 March to 1 April 2007 (CR 2054). At this time the STEREO observatories were separated by only 3 degrees in heliographic longitude, and less than 1 degree in heliographic latitude. 


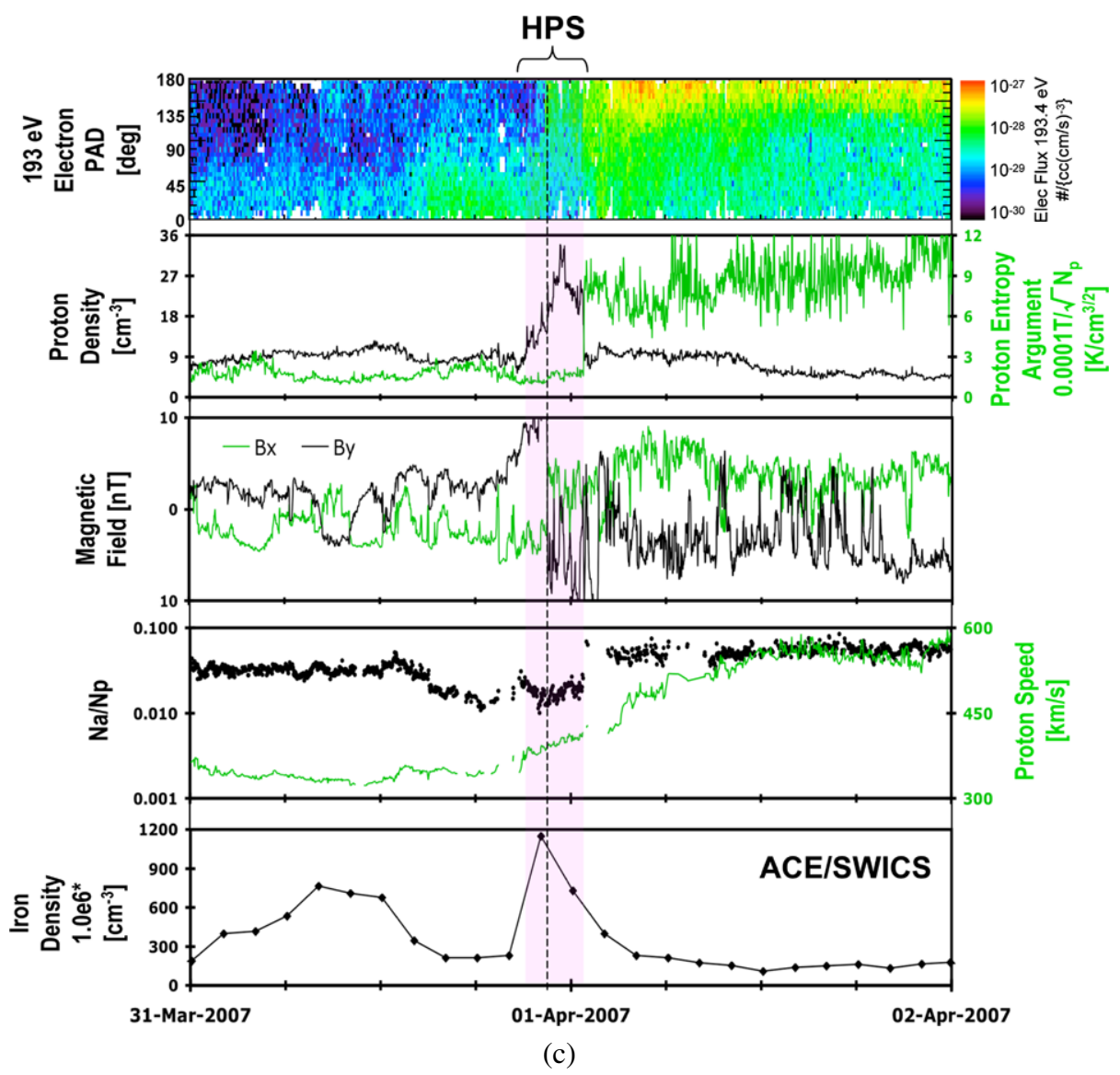

Figure 2 (Continued.)

The radial separation between ST-A and ST-B was about $0.06 \mathrm{AU}$, or $8.0 \times 10^{6} \mathrm{~km}$. At these scales, a solar wind feature that is roughly parallel to the ideal Parker spiral angle of 45 degrees and traveling away from the Sun is expected to arrive at Wind first, ST-A second, and ST-B third. This is illustrated in Figure 3. The start times listed in Table 1 agree with this expectation. As the HPS passed over the respective spacecraft, each observed an increase in proton density, from $8 \mathrm{~cm}^{-3}$ to a peak of about $30 \mathrm{~cm}^{-3}$. The density dropped back to $8 \mathrm{~cm}^{-3}$ following the passage of the HPS. ST-A/PLASTIC observed an increase in iron ion density from about $250 \times 10^{-6} \mathrm{~cm}^{-3}$ to $750 \times 10^{-6} \mathrm{~cm}^{-3}$. The iron density then returned to $250 \times$ $10^{-6} \mathrm{~cm}^{-3}$ at ST-A. The iron densities at ACE and ST-B were similarly enhanced when encountering the HPS, with the absolute density at ACE increasing from $230 \times 10^{-6} \mathrm{~cm}^{-3}$ to $1150 \times 10^{-6} \mathrm{~cm}^{-3}$. During this time the solar wind proton speed remained fairly steady at the respective spacecraft. Based on the observed speeds and the crossing times, the HPS was found to have a radial width of between $5.0 \pm 0.1 \times 10^{6} \mathrm{~km}(0.033 \pm 0.001 \mathrm{AU})$ and $6.7 \pm 0.2 \times 10^{6} \mathrm{~km}(0.045 \pm 0.001 \mathrm{AU})$. The HPS was narrowest at Wind and widest at ST-A. 


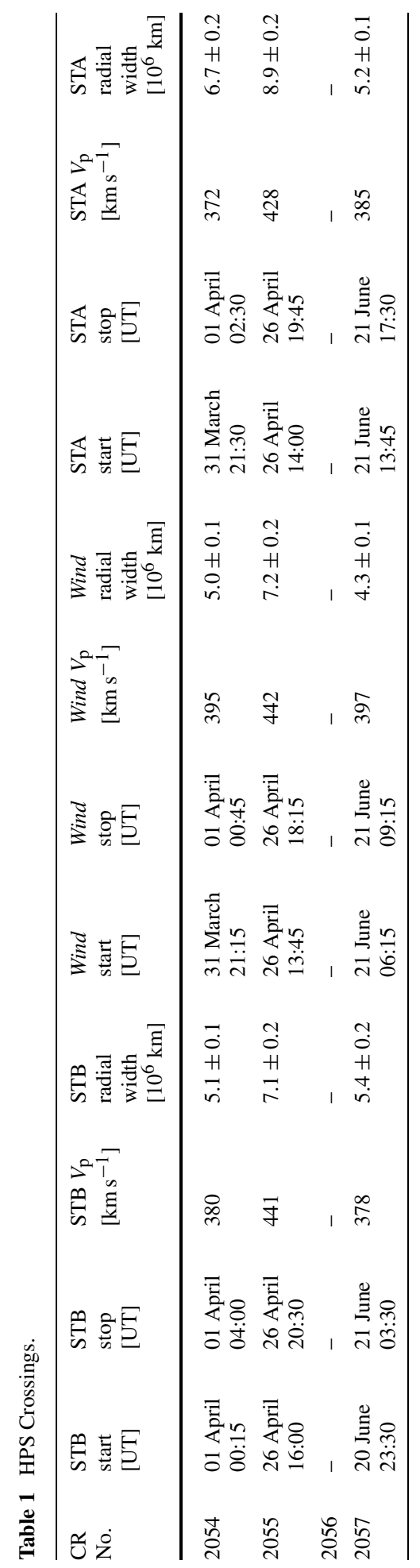


Figure 3 Locations of the three spacecraft on 31 March 2007. A segment of the idealized Parker spiral is shown as a light brown line crossing the panel at 45 degrees. This demonstrates how the HPS (assumed parallel to the Parker spiral) could cross the Wind spacecraft, followed by ST-A and then ST-B.

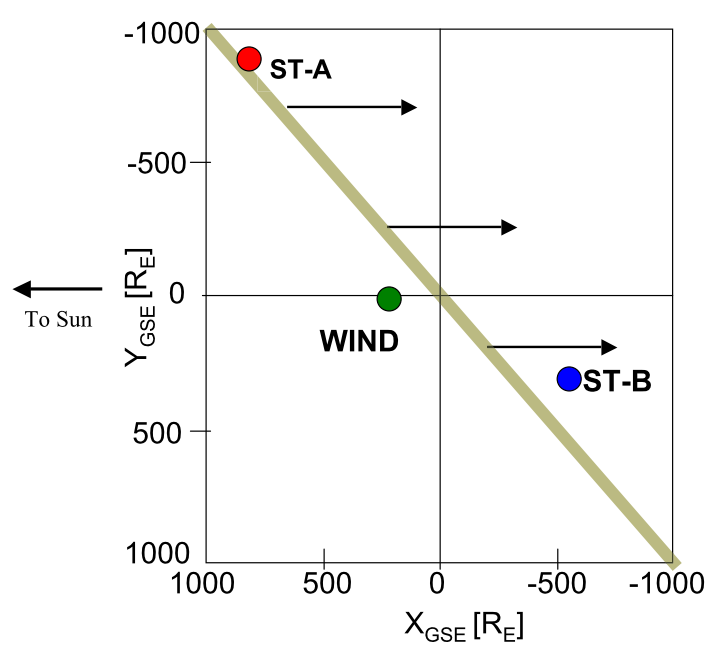

\subsection{CROSSING 2, CR 2055}

The second HPS crossing (see Figures 4a, 4b, and 4c) took place on 26 April 2007 (CR 2055). The STEREO observatories were separated by 5.5 degrees in heliographic longitude, and by $0.08 \mathrm{AU}$ in heliocentric radial distance. Like the first case, the HPS is expected to traverse Wind, then ST-A, and lastly ST-B. The proton density enhancement was more modest than the first crossing, increasing from about $7 \mathrm{~cm}^{-3}$ to $16 \mathrm{~cm}^{-3}$. The iron density at ST-A slowly increased from about $200 \times 10^{-6} \mathrm{~cm}^{-3}$ to $375 \times 10^{-6} \mathrm{~cm}^{-3}$. The enhancement is less pronounced at ACE with an increase from about $200 \times 10^{-6} \mathrm{~cm}^{-3}$ to $285 \times 10^{-6} \mathrm{~cm}^{-3}$. The iron density at ST-B shows a similar trend. Again the speed was fairly constant throughout the HPS, but at 430 to $440 \mathrm{~km} \mathrm{~s}^{-1}$ the observed speed was faster than during the first crossing. The observed HPS widths ranged from about $7.1 \pm 0.2 \times 10^{6} \mathrm{~km}(0.047 \pm 0.001 \mathrm{AU})$ to $8.9 \pm 0.2 \times 10^{6} \mathrm{~km}(0.059 \pm 0.001 \mathrm{AU})$.

\subsection{CROSSING 3, CR 2056}

The sector boundary crossing during CR 2056 was modified by the passage of magnetic clouds associated with eruptions from active region 10956. Kilpua et al. (2009) identified two magnetic clouds encountered by ST-A during 22-23 May 2007, while ST-B and Wind encountered one magnetic cloud during this period. Based on Grad-Shafronov reconstruction, Kilpua et al. (2009) found ST-B and Wind crossed near the center of the first cloud, while ST-A received just a glancing blow. The second cloud delivered a much more direct impact to ST-A (Möstl et al., 2009), and had a slight encounter with Wind, but missed ST-B. (See Figures 1 and 6 of Kilpua et al., 2009.)

Assuming an equatorial, sidereal, solar angular rotation speed of 14.38 degrees per day (Newton and Nunn, 1951) and taking into account the motion of the spacecraft, the third HPS crossing was expected to take place on 23 May 2007, when the STEREO observatories were separated by about 9 degrees in heliographic longitude and $0.1 \mathrm{AU}$ in radial distance from the Sun.

Figures 5 and 6 present the in situ ST-B and ST-A data (respectively) for the time period in which the third HPS crossing was expected to occur, with a day or so of margin shown on each side. Crooker, Gosling, and Kahler (1998) and Crooker et al. (1998) studied magnetic 


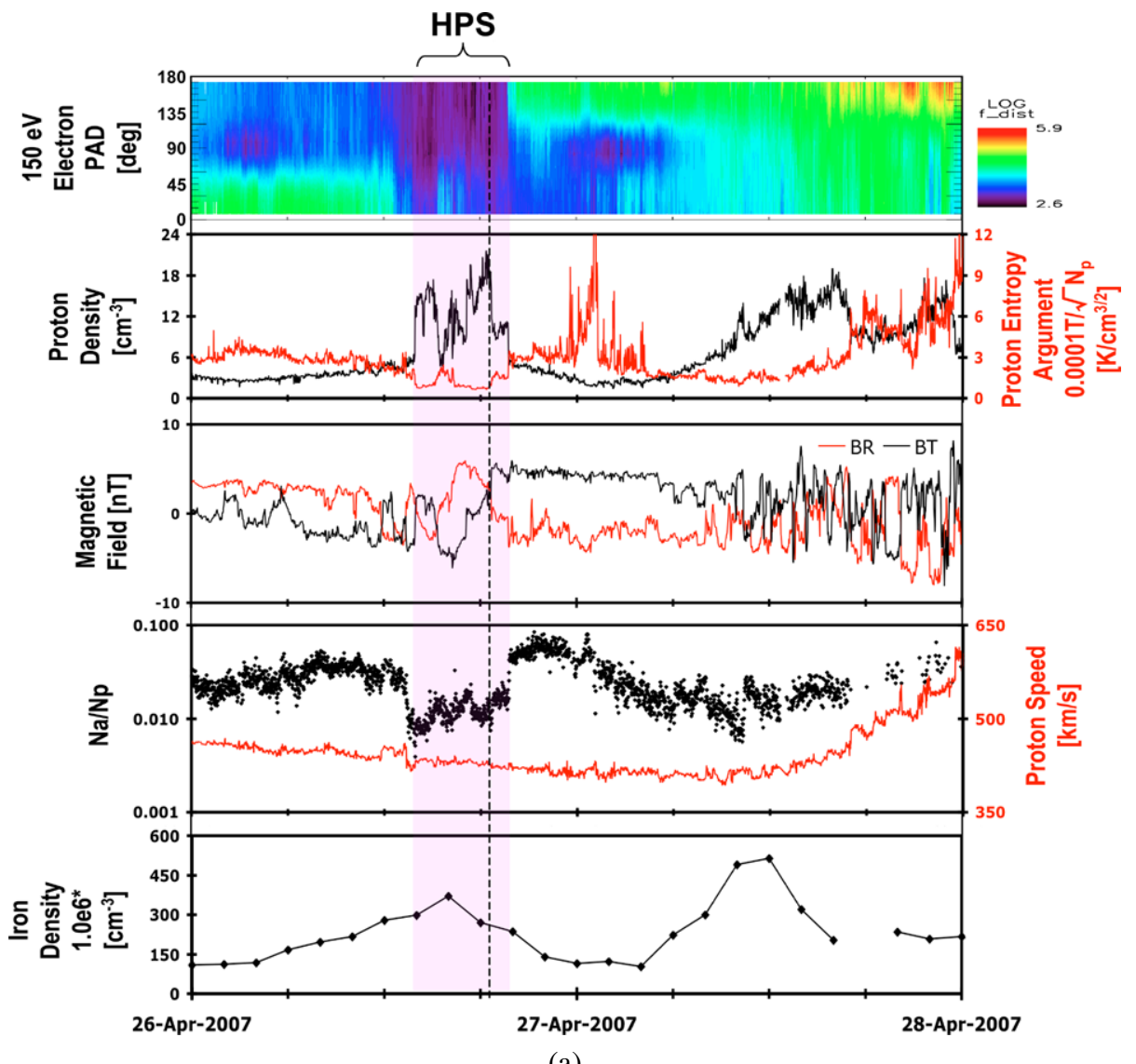

(a)

Figure 4 (a) ST-A in situ data from the second studied HPS crossing. From top to bottom the panels are: $150 \mathrm{eV}$ electron PADs, proton density (black) and proton entropy argument (red), magnetic field components $\mathrm{BR}$ (red) and BT (black), $N_{\mathrm{a}} / N_{\mathrm{p}}$ (black) and solar wind proton speed (red), and iron ion density. The magenta shaded region near the center is the HPS. The dashed vertical line is the HCS. (b) ST-B in situ data from the second studied HPS crossing. From top to bottom the panels are: $150 \mathrm{eV}$ electron PADs, proton density (black) and proton entropy argument (blue), magnetic field components BR (blue) and BT (black), and $N_{\mathrm{a}} / N_{\mathrm{p}}$ (black) with solar wind proton speed (blue), and iron ion density. The magenta shaded region near the center is the HPS. The dashed vertical line is the HCS. (c) Wind in situ data from the second studied HPS crossing. From top to bottom the panels are: $193 \mathrm{eV}$ electron PADs, proton density (black) and proton entropy argument (green), magnetic field components $B_{x}$ (green) and $B_{y}$ (black), and $N_{\mathrm{a}} / N_{\mathrm{p}}$ (black) with solar wind proton speed (green). The bottom panel shows the iron ion density from ACE/SWICS. The magenta shaded region near the center is the HPS. The dashed vertical line is the HCS.

clouds at sector boundaries, and found that the magnetic cloud can cause the sector boundary to arrive early, thus the need for our margin. The magnetic clouds identified by Kilpua et al. (2009) have been shaded in grey and labeled as MC 1 (magnetic cloud 1) and MC 2 (magnetic cloud 2) in Figures 5 and 6. Kilpua et al. (2009) identifies the sector boundary crossings at both ST-A and ST-B on 24 May based upon the reversal of the electron heat flux distribution. No clear HPS could be identified that met all of our criteria. 


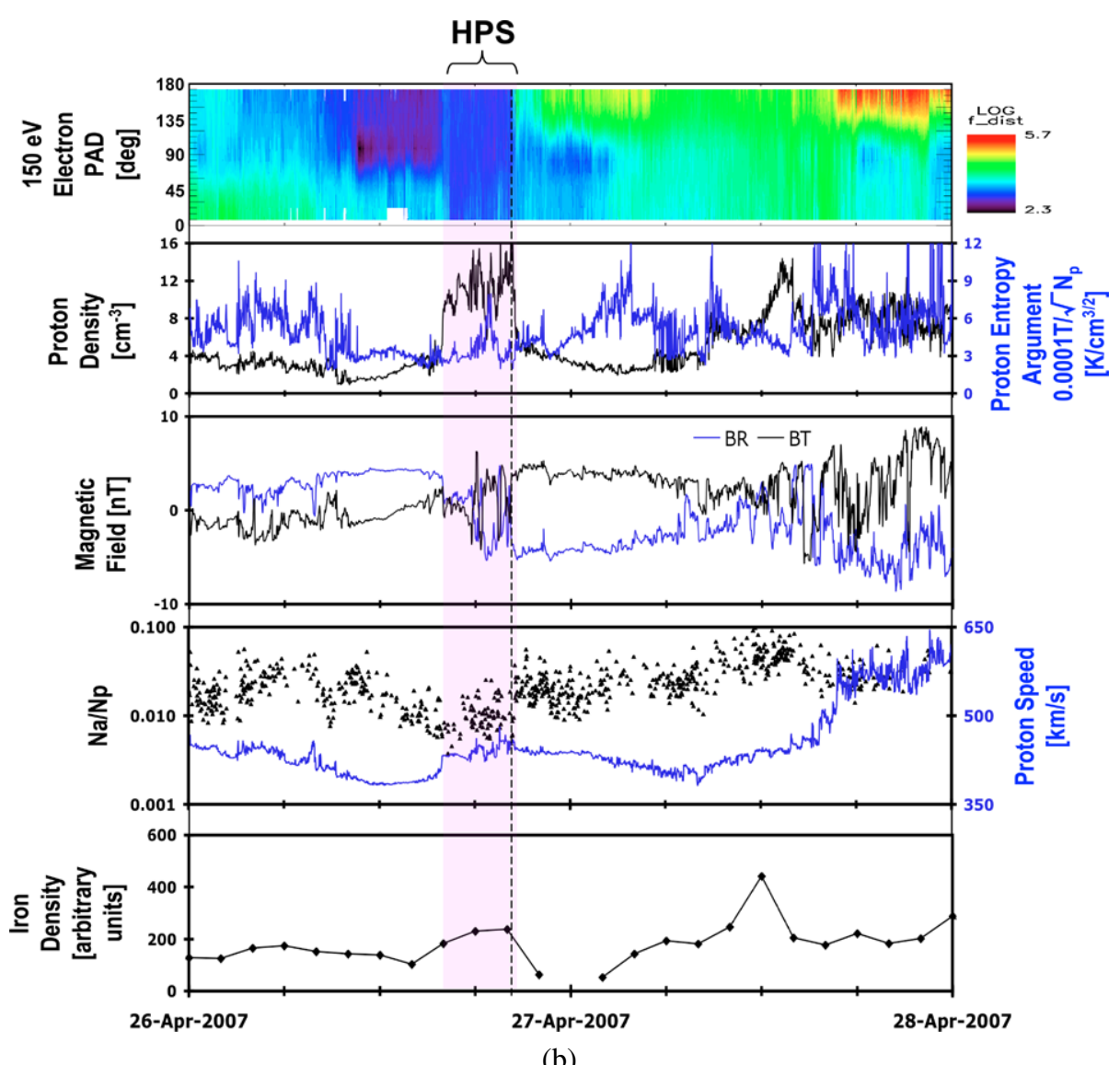

Figure 4 (Continued.)

\subsection{CROSSING 4, CR 2057}

The final crossings of the HPS (see Figures $7 \mathrm{a}, 7 \mathrm{~b}$, and 7c) in the study period took place on 20 and 21 June 2007 (CR 2057). The STEREO observatories were separated by about 14 degrees in heliographic longitude, and $0.12 \mathrm{AU}$ in orbital radii. The longitude separation was dominant over the radial separation for a structure lying along the 45 degree ideal Parker spiral, and the HPS arrival order agreed with expectation: ST-B, followed by Wind, and finally ST-A. Unlike the first two crossings, the proton density profiles are noticeably different between the three observatories. ST-B saw a moderate change from $\sim 2 \mathrm{~cm}^{-3}$ to $6 \mathrm{~cm}^{-3}$. Wind observed a more extreme range of $4.5 \mathrm{~cm}^{-3}$ to $30 \mathrm{~cm}^{-3}$. ST-A observed an intermediate range of about $4.5 \mathrm{~cm}^{-3}$ to $15 \mathrm{~cm}^{-3}$. The speed profiles at ST-A and ST-B are essentially flat while the HPS is crossing over them, but there is an increase in speed at Wind. The iron data from ST-A show the density increases from about $150 \times 10^{-6} \mathrm{~cm}^{-3}$ to $250 \times 10^{-6} \mathrm{~cm}^{-3}$. At ACE the iron density increased from about $70 \times 10^{-6} \mathrm{~cm}^{-3}$ to $260 \times 10^{-6} \mathrm{~cm}^{-3}$. Again, ST-B also observes an enhancement in iron density. The radial width of the HPS ranged from $4.3 \pm 0.1 \times 10^{6} \mathrm{~km}(0.029 \pm 0.001 \mathrm{AU})$ to $5.4 \pm 0.2 \times 10^{6} \mathrm{~km}$ $(0.036 \pm 0.001 \mathrm{AU})$. 


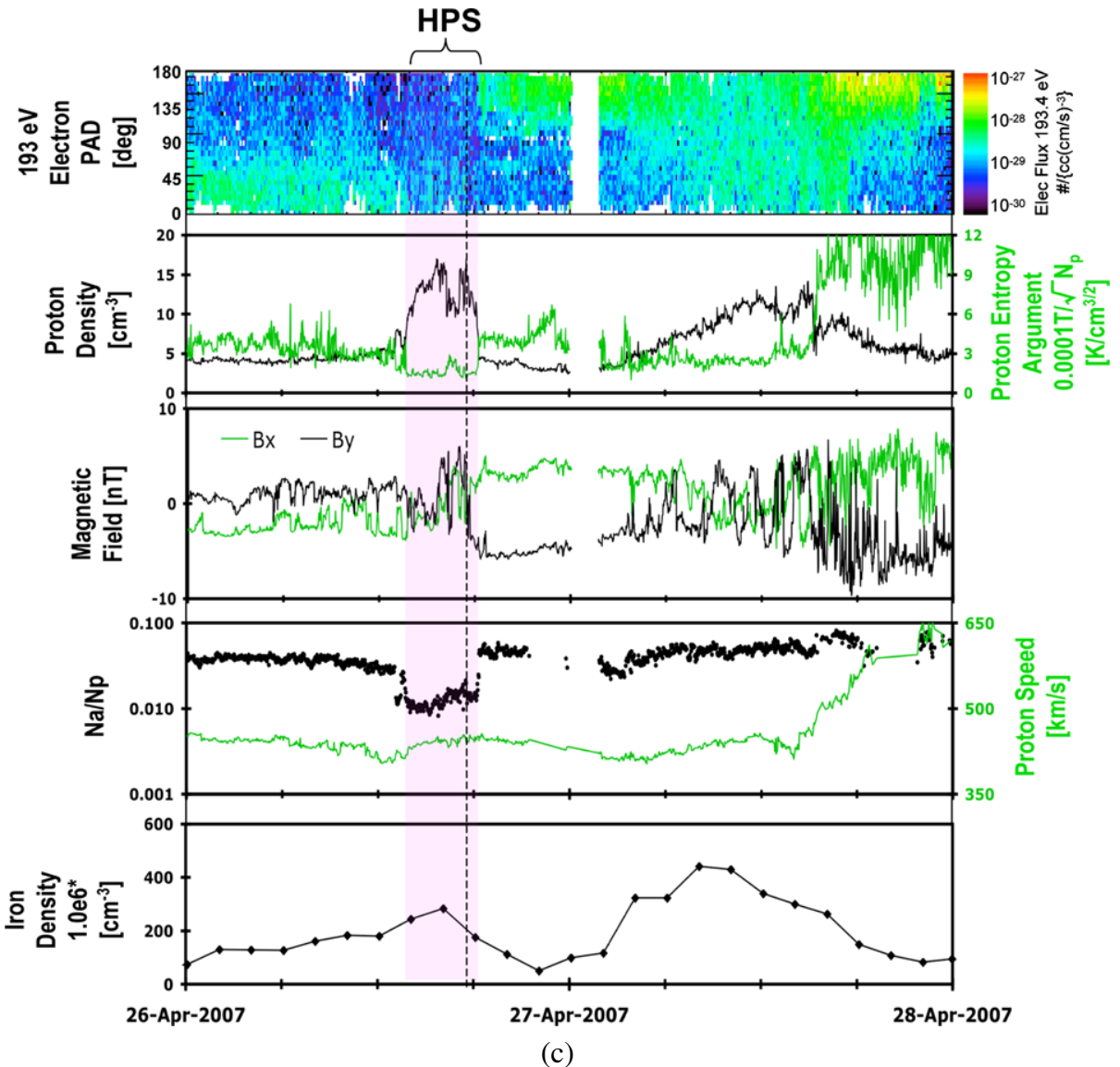

Figure 4 (Continued.)

\section{Summary and Discussion}

We have combined in situ observations from three in-ecliptic spacecraft slowly separating in heliographic longitude to study successive crossings of the HPS over four solar rotations. This study is unique in its multiple vantage points for each crossing of the HPS. We can therefore compare plasma parameters not only from one solar rotation to the next, but within the same Carrington rotation over time scales of hours. We have chosen several questions to address with this data set. First, can the HPS be better described as transient ( $c f$. Suess et al., 2009) or quasi-stationary (cf. Liu et al., 2010b) in nature? Second, what is the origin of disparities in the HPS properties from one spacecraft to another when the temporal separation between observations is much less than a Carrington rotation? Third, what can we learn about the solar source region of the HPS?

To begin addressing the first question we infer the in-ecliptic geometry of the HPS in the vicinity of the spacecraft for the three Carrington rotations without magnetic clouds (CR 2054, 2055, and 2057). Each panel in Figure 8 shows the locations of the three spacecraft in the ecliptic plane at a time when Wind encounters the leading edge of the HPS. The radial (i.e. along the Sun-spacecraft line) width of the HPS at each spacecraft was calculated 


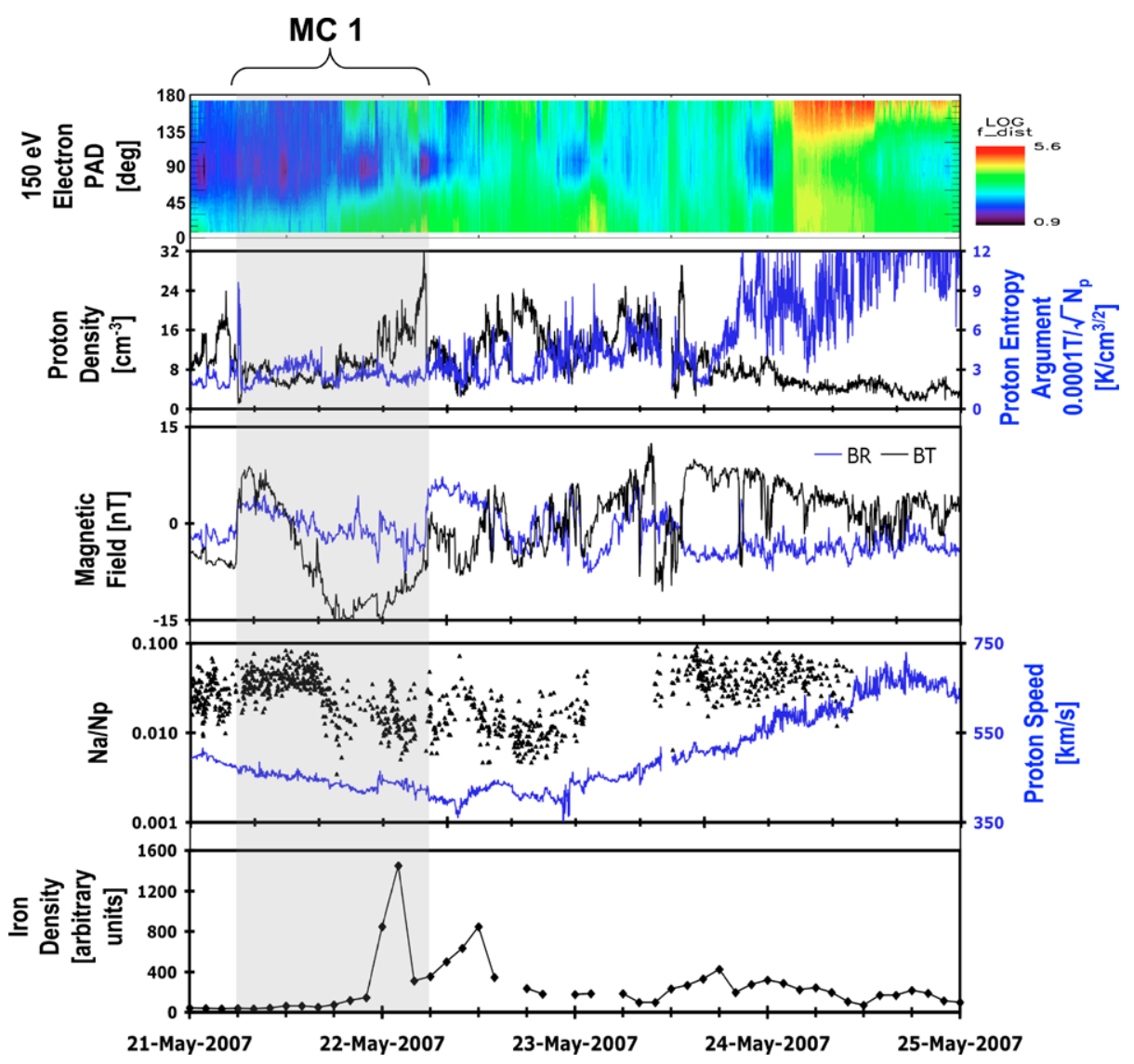

Figure 5 ST-B in situ data from the period in which a HPS crossing was expected in CR No. 2056. Magnetic cloud 1 (MC 1) as identified by Kilpua et al. (2009) has been shaded in grey.

using the in situ speeds and start/end times. These widths are shown in Figure 8 as grey lines. Curves have been added to guide the eye and visualize the leading (violet) and trailing (green) edges. Figure 8 shows that the HPS lies roughly parallel to the ideal Parker spiral (light blue curve) in the ecliptic plane for the three cases not disturbed by ICMEs. The Parker spiral curve shown in each panel is based upon the average measured solar wind speed during the HPS passage at Wind. The widths vary from spacecraft to spacecraft within each Carrington rotation $(2054,2055$, and 2057) in a way that is inversely proportional to the average measured solar wind speed. We interpret this as compression resulting from interaction with the fast stream following the HPS.

Foullon et al. (2009) studied the same HPS crossing in CR 2053 and found the width along the Sun-Earth line to be $560-580 R_{\mathrm{E}}$, or $3.6-3.7 \times 10^{6} \mathrm{~km}$. The average width from the three observations in CR 2054 is $5.6 \times 10^{6} \mathrm{~km}$, and in CR 2055 it is $7.7 \times 10^{6} \mathrm{~km}$. In other words, the radial extent of the HPS is growing from one CR to another before it is disrupted by the ICME in CR 2056.

Suess et al. (2009) suggest that the HPS is created through the transient release of plasma "blobs" with low $N_{\mathrm{a}} / N_{\mathrm{p}}$ from streamer cores. They further propose that the blobs are split by flow shears, resulting in the HCS being observed on one edge of the HPS, rather than 


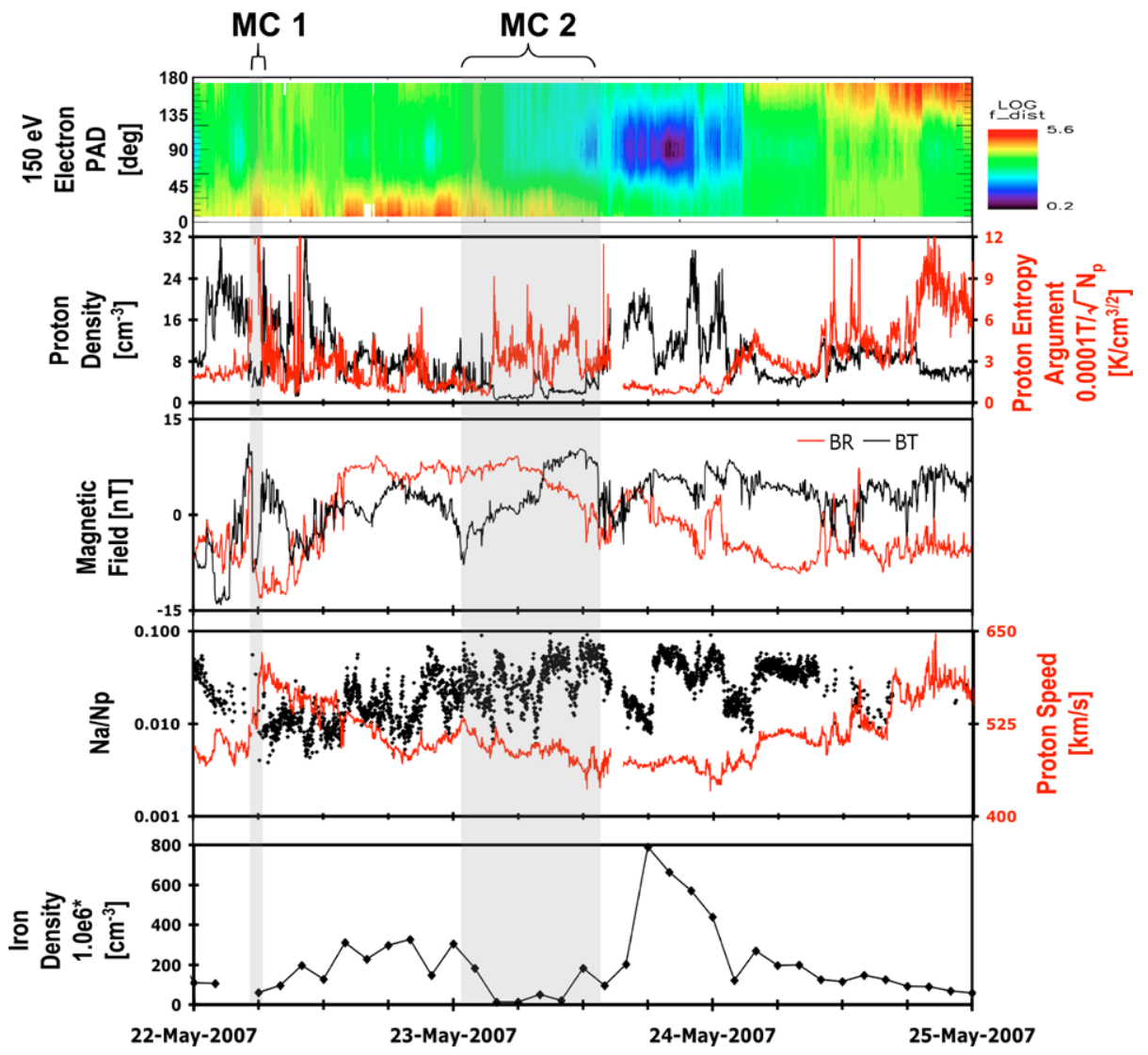

Figure 6 ST-A in situ data from the period in which a HPS crossing was expected in CR No. 2056. Magnetic clouds 1 and 2 (MC 1 and MC 2) as identified by Kilpua et al. (2009) have been shaded in grey.

in the center of the HPS. (See Figure 15 of Suess et al., 2009.) In this scenario we would expect multi-point observations within the same solar rotation to show some cases with the HCS at the leading edge of the HPS, and others at the trailing edge. Figure 9 shows the proton density plotted against time for all three spacecraft during the HPS crossings of CR 2054 (top panel), 2055 (middle panel), and 2057 (bottom panel). The regions identified as the HPS at each spacecraft have been shaded. The vertical black lines indicate the location of the HCS. In CR 2054 the HPS straddles the HCS, with more HPS material following the HCS than preceding it. In CR 2055 and 2057 the situation is reversed, with most of the HPS preceding the HCS. Figure 9 shows that the location of the HCS within the HPS is similar from spacecraft to spacecraft within each Carrington rotation. The HPS appears to be quasi-stationary (rather than transient) on time scales of a day or less, consistent with Liu et al. (2010b). At the same time, the HPS is clearly changing from one solar rotation to another. In other words, the HPS is quasi-stationary for a day (or longitudinal spatial scales of 14 degrees), but less than a Carrington rotation. A follow-up study with multi-spacecraft observations at larger temporal (spatial) separations is planned to narrow this range.

Our second topic is differences in observed HPS properties over time scales much less than a Carrington rotation. Looking again at the density profiles in Figure 9, they are similar 


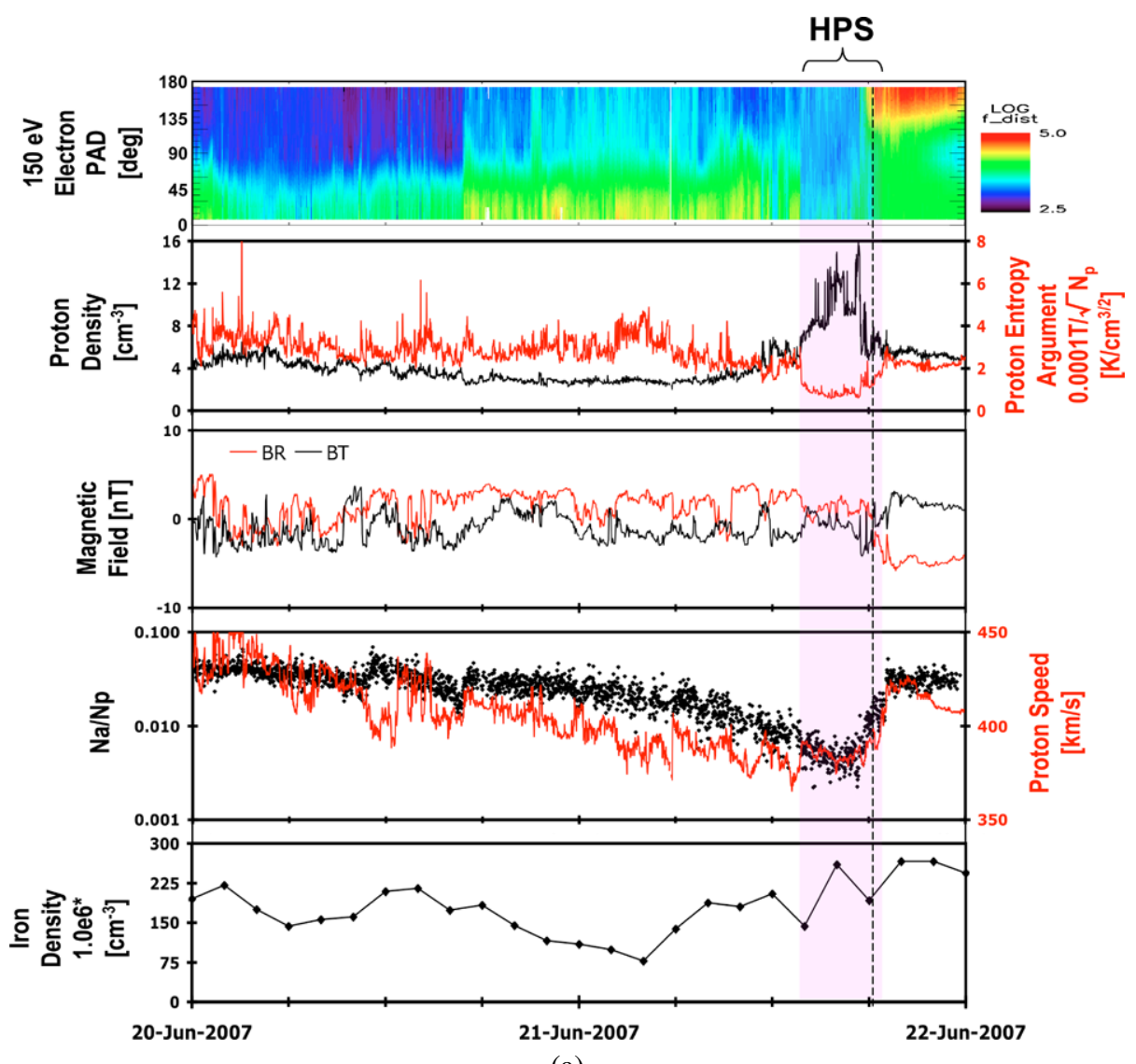

(a)

Figure 7 (a) ST-A in situ data from the fourth studied HPS crossing. From top to bottom the panels are: $150 \mathrm{eV}$ electron PADs, proton density (black) and proton entropy argument (red), magnetic field components $\mathrm{BR}$ (red) and BT (black), $N_{\mathrm{a}} / N_{\mathrm{p}}$ (black) and solar wind proton speed (red), and iron ion density. The magenta shaded region near the center is the HPS. The dashed vertical line is the HCS. (b) ST-B in situ data from the fourth studied HPS crossing. From top to bottom the panels are: $150 \mathrm{eV}$ electron PADs, proton density (black) and proton entropy argument (blue), magnetic field components BR (blue) and BT (black), and $N_{\mathrm{a}} / N_{\mathrm{p}}$ (black) with solar wind proton speed (blue), and iron ion density. The magenta shaded region near the center is the HPS. The dashed vertical line is the HCS. (c) Wind in situ data from the fourth studied HPS crossing. From top to bottom the panels are: $193 \mathrm{eV}$ electron PADs, proton density (black) and proton entropy argument (green), magnetic field components $B_{x}$ (green) and $B_{y}$ (black), and $N_{\mathrm{a}} / N_{\mathrm{p}}$ (black) with solar wind proton speed (green). The bottom panel shows the iron ion density from ACE/SWICS. The magenta shaded region near the center is the HPS. The dashed vertical line is the HCS.

between the three spacecraft for CR 2054 and 2055. The density enhancements are about the same in magnitude, though small-scale structures within the enhancements are certainly not identical. The bottom panel in Figure 9 is the density profile for CR No. 2057. On 21 June 2007 the heliographic longitude separation between ST-A and ST-B was about 14 degrees. The HPS was encountered by ST-A about 14 hours after its arrival at ST-B. The density profiles are clearly not in agreement between the three spacecraft. The density enhancement is by far the greatest at Wind. The density profile at ST-A is lower by a factor of about 2.5, and lower by yet another factor of 2 at ST-B. The large rise in density at Wind, not present 


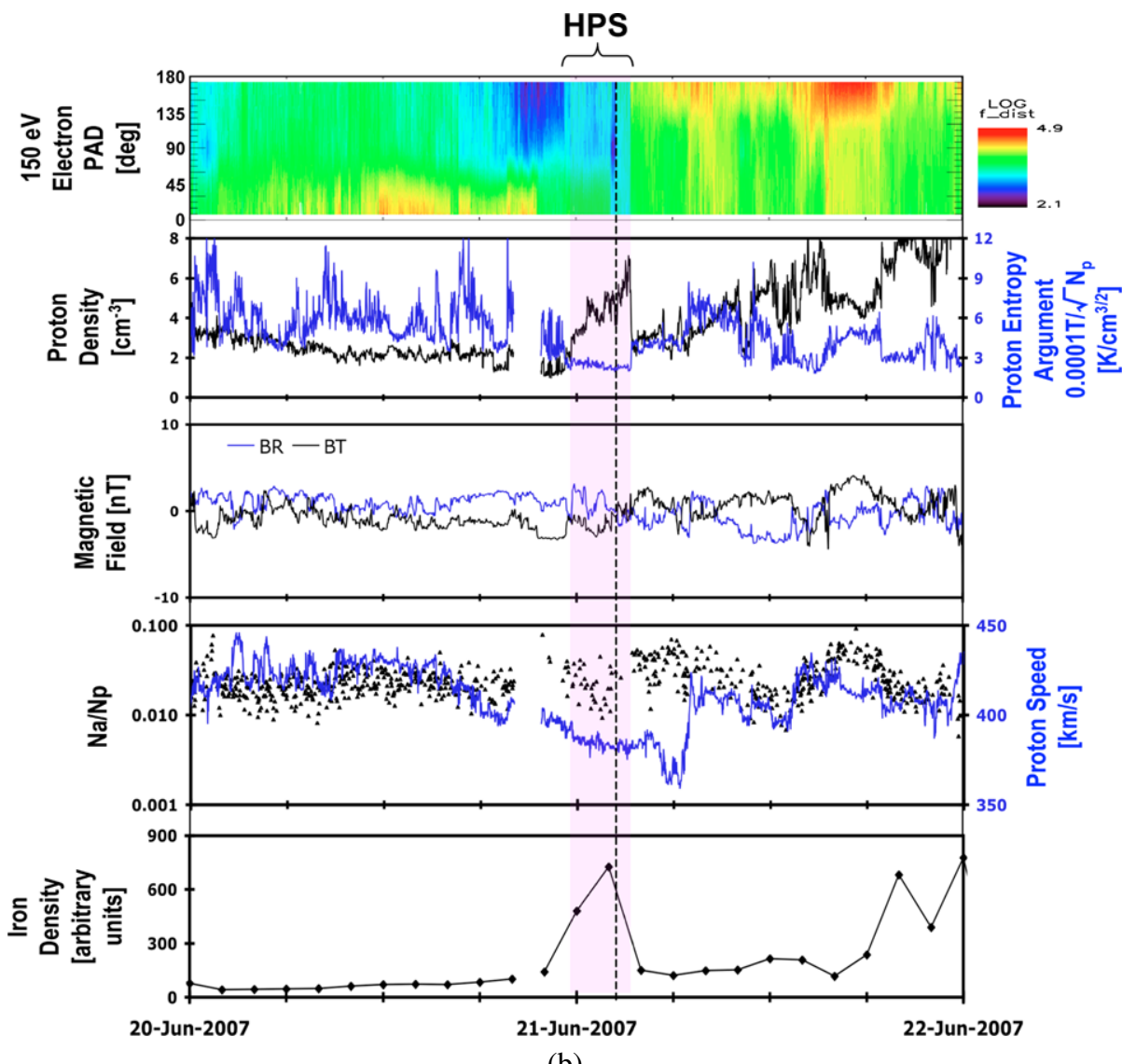

Figure 7 (Continued.)

at the other spacecraft, is particularly interesting given that Wind was located between ST-B and ST-A in both space and time. Figure 10 shows that the speed profiles at ST-B and ST-A are more or less flat in the vicinity of the HPS, while the solar wind speed at Wind was undergoing a gradual increase. The duration of the HPS crossing at Wind was also shorter than at ST-A and ST-B. The difference in density and duration, combined with the increasing speed observed at Wind suggest a developing compression region due to the interaction of slow and fast streams. In fact, the end boundary of the HPS at Wind also meets the definition of a stream interface (SI): an abrupt drop in density accompanied by an increase in speed and temperature and a westward flow deflection (Burlaga, 1974; Gosling et al., 1978).

The top panel of Figure 11 shows the ST-A/SECCHI/EUV synoptic map for CR 2057. The HPS crossing of interest is indicated with a light blue circle, and the coronal hole from which the fast stream encountered by Wind most likely originates is highlighted in green. The bottom left-hand panel in Figure 11 is a synoptic map created from STA/SECCHI/COR1 images at $2.2 R_{\text {Sun }}$. About one fourth of Carrington Rotation 2057 is shown. The nearly horizontal red, green, and blue traces are projections of the ST-A, Wind, and ST-B spacecraft trajectories. The heliographic latitude separation between the three spacecraft is about 2 degrees, with ST-A and ST-B bracketing Wind. The light blue cir- 


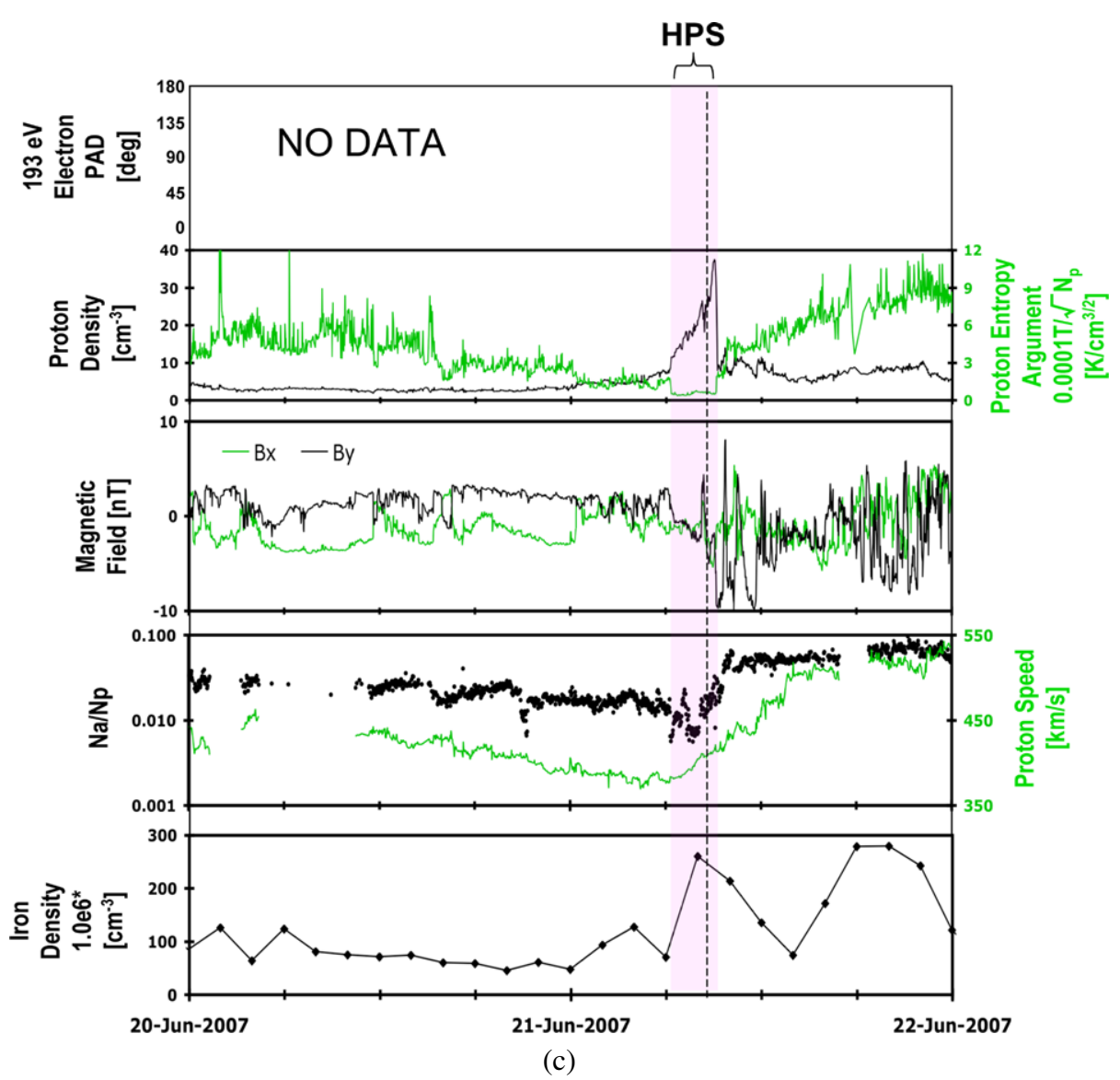

Figure 7 (Continued.)

cle indicates the region associated with the CR 2057 HPS crossing, while the outline of the coronal hole is shown in green. The SECCHI synoptic map shows both a streamer associated with the HCS and a pseudo-streamer (which does not mark a change in magnetic sector; $c f$. Wang, Sheeley, and Rich, 2007). Both the streamer and pseudo-streamer are potential sources of slow solar wind. The lower right-hand panel of Figure 11 shows the solar wind speed obtained from the WSA model using magnetograms from the Wilcox Solar Observatory as input. The WSA model output shows slow solar wind originating from both the streamer and the pseudo-streamer in Carrington rotation 2057. As there are no obvious changes in the source region (based upon imaging observation), the most likely explanation for the differences in speed and density between the three spacecraft is that Wind connects to the fast wind from a coronal hole at a Carrington longitude where ST-B and ST-A are encountering material from the streamer belt and/or the pseudo-streamer. Connection to different source regions is possible with the small heliographic latitude separation between the spacecraft (Schwenn, 1990).

The final topic we address is what we can learn about the solar source region of the HPS. We observed changes in composition coincident with the HPS, setting it apart from the adjacent non-HPS slow solar wind. This is shown in Figures 12a, 12b, and 12c. In each 
Figure 8 The locations of the spacecraft are shown for the HPS crossings observed during Carrington rotations 2054, 2055, and 2057. (The crossing during Carrington rotation 2056 was disrupted by the passage of magnetic clouds.) The red diamond is ST-A, the green circle is Wind, and the blue triangle is ST-B. The measured radial widths are shown as grey lines, while the boundaries have been connected with purple and green traces. An ideal Parker spiral is shown in light blue based upon the average solar wind speed measured in situ by Wind/SWE.
HPS Crossing CR No. 2054

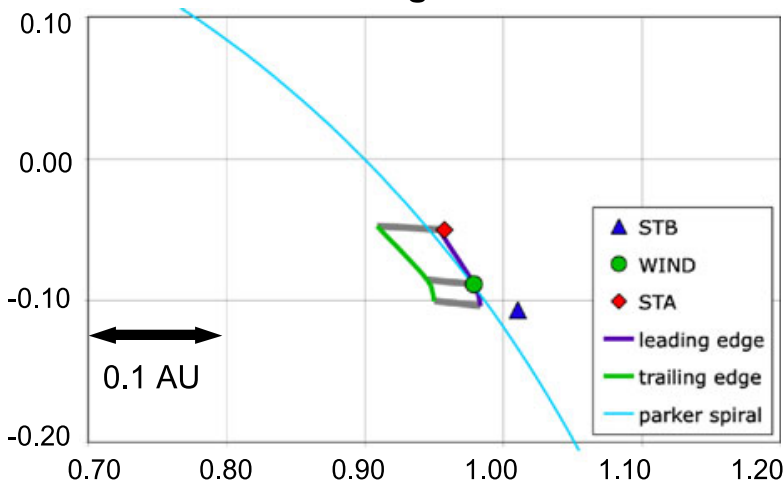

HPS Crossing CR No. 2055

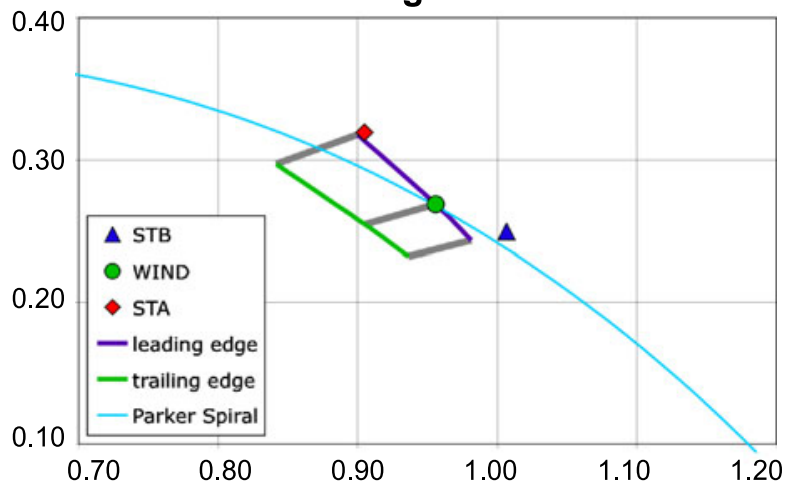

HPS Crossing CR No. 2057

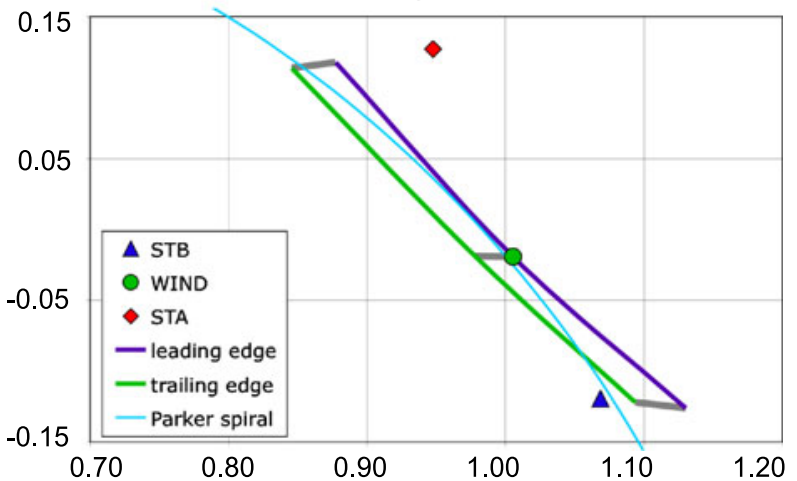

panel the black trace is the proton density, red is alpha density, and green is iron density. The alpha and iron densities have been scaled for easier comparison with the protons. It should again be noted that the iron densities shown for ST-B (Figure 12b) are in terms of arbitrary units. Figure 12c shows ion data from the L1 spacecraft ACE because iron densities are not available from Wind. The proton densities shown in Figure 12c are from ACE SWEPAM (McComas et al., 1998), while helium and iron densities are from ACE SWICS (Gloeckler et al., 1998). In all panels of Figures 12a, 12b, and 12c the identified 


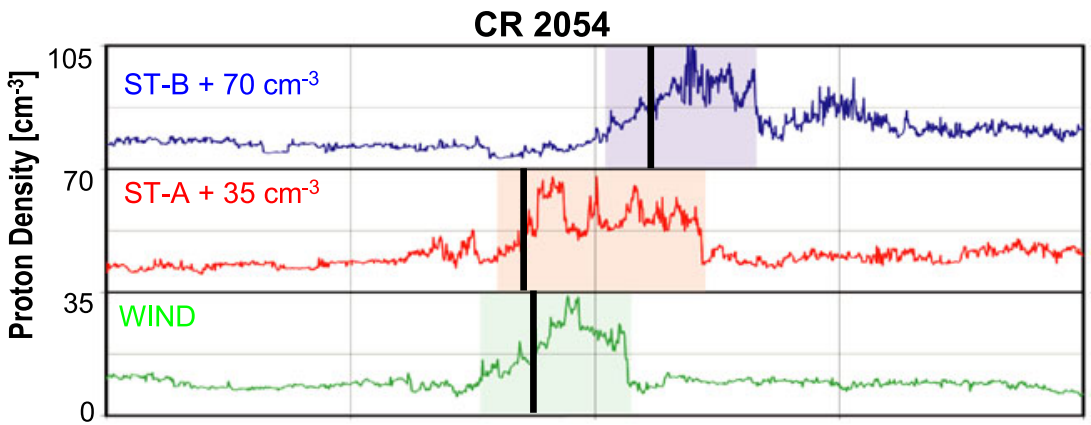

31-Mar-2007

01-Apr-2007

$12: 00$

CR 2055

12:00

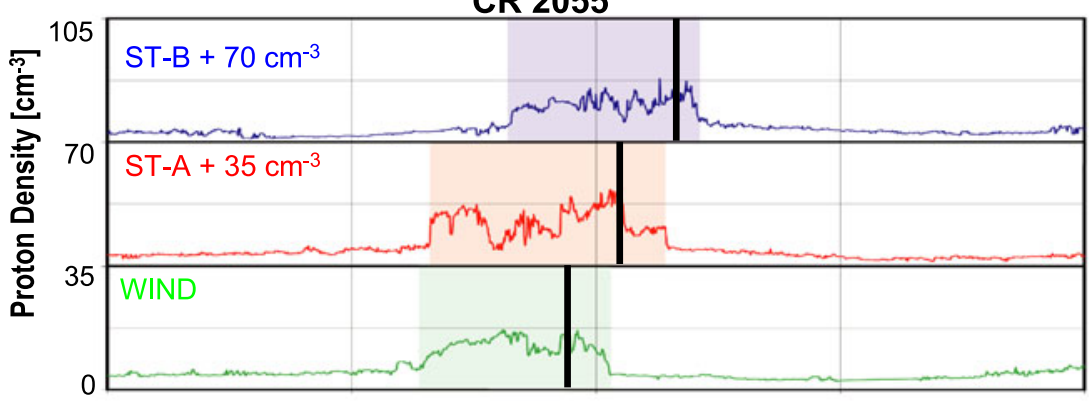

26-Apr-2007

27-Apr-2007

06:00

CR 2057

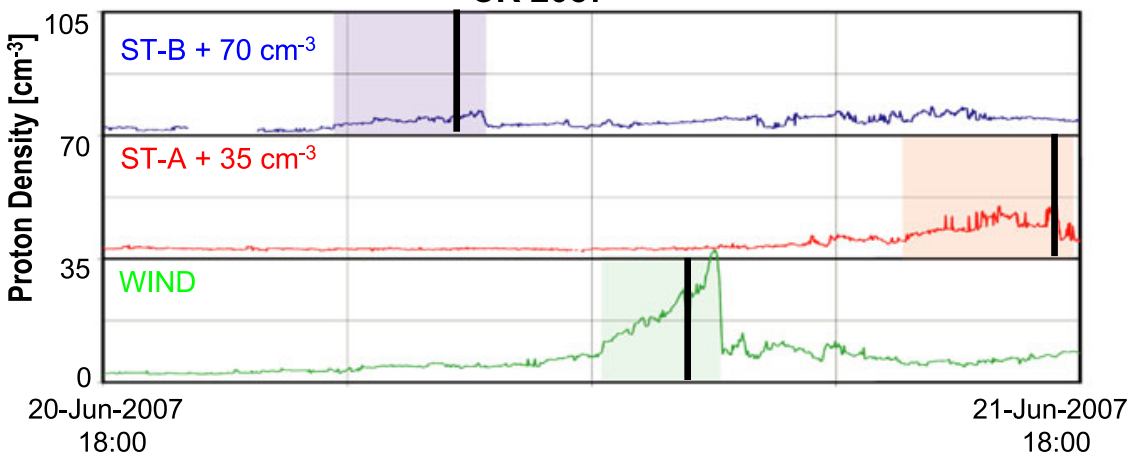

Figure 9 Solar wind proton densities at ST-B (blue), ST-A (red), and Wind (green) during CR 2054, 2055, and 2057. The HPS crossing at each spacecraft is indicated with shading. Vertical lines indicate the position of the HCS.

HPS has been shaded. The first compositional signature of the HPS is an increase in proton density, one of our required criteria for identification. We found that alpha number density (in red) shows little if any depletion in the HPS compared to the adjacent regions, so the change in the alpha to proton density ratio is attributed primarily to the increase in protons. All three spacecraft (ST-A, ST-B, and ACE) observed a modest increase in the density of iron ions (green traces) coincident with the HPS, consistent with observations presented by Schmid, Bochsler, and Geiss (1988). CR 2054, 2055, and 2057 were free of transients, so we do not attribute the increase in iron density to remnants of ICMEs. The enhancement in iron 


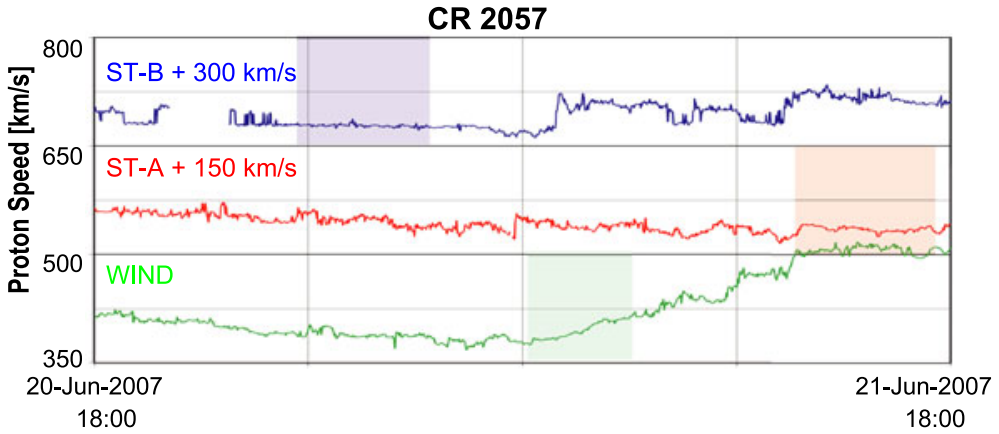

Figure 10 Solar wind proton speed at ST-B (blue), ST-A (red), and Wind (green) during the CR 2057 HPS crossing. The HPS crossing at each spacecraft is indicated with shading.

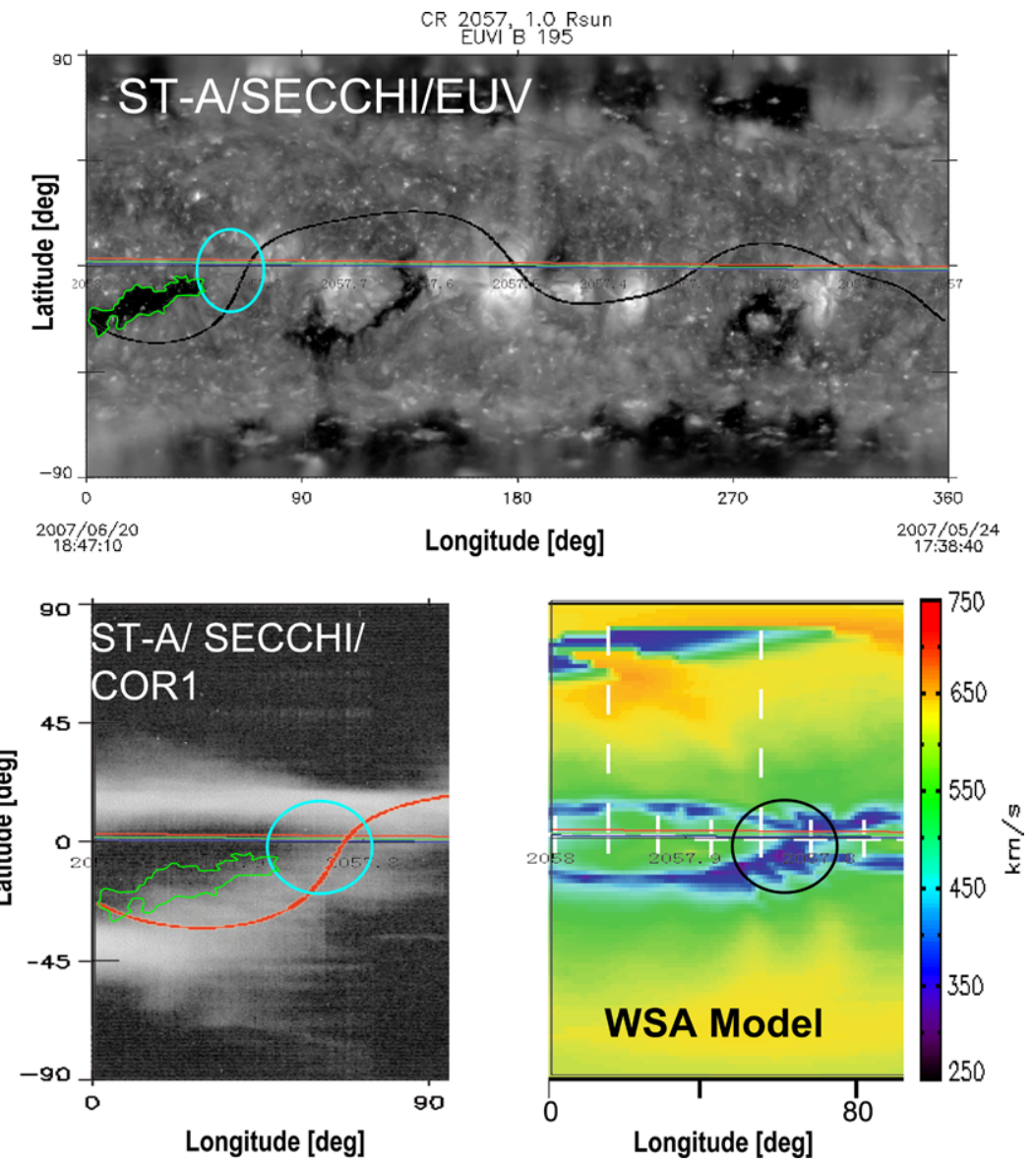

Figure 11 Top panel: Same format as Figure 1, CR 2057. The coronal hole outlined in green is the most likely source of the fast stream observed by Wind. Bottom-left panel: Modeled HCS overlaid on a synoptic map of the white-light corona at $2.2 R_{\text {Sun }}$. The outline of the same coronal hole as the top panel is shown in green. Bottom-right panel: Expected solar wind speed from the output of the WSA model for the same longitude region as shown in the bottom-left panel. 

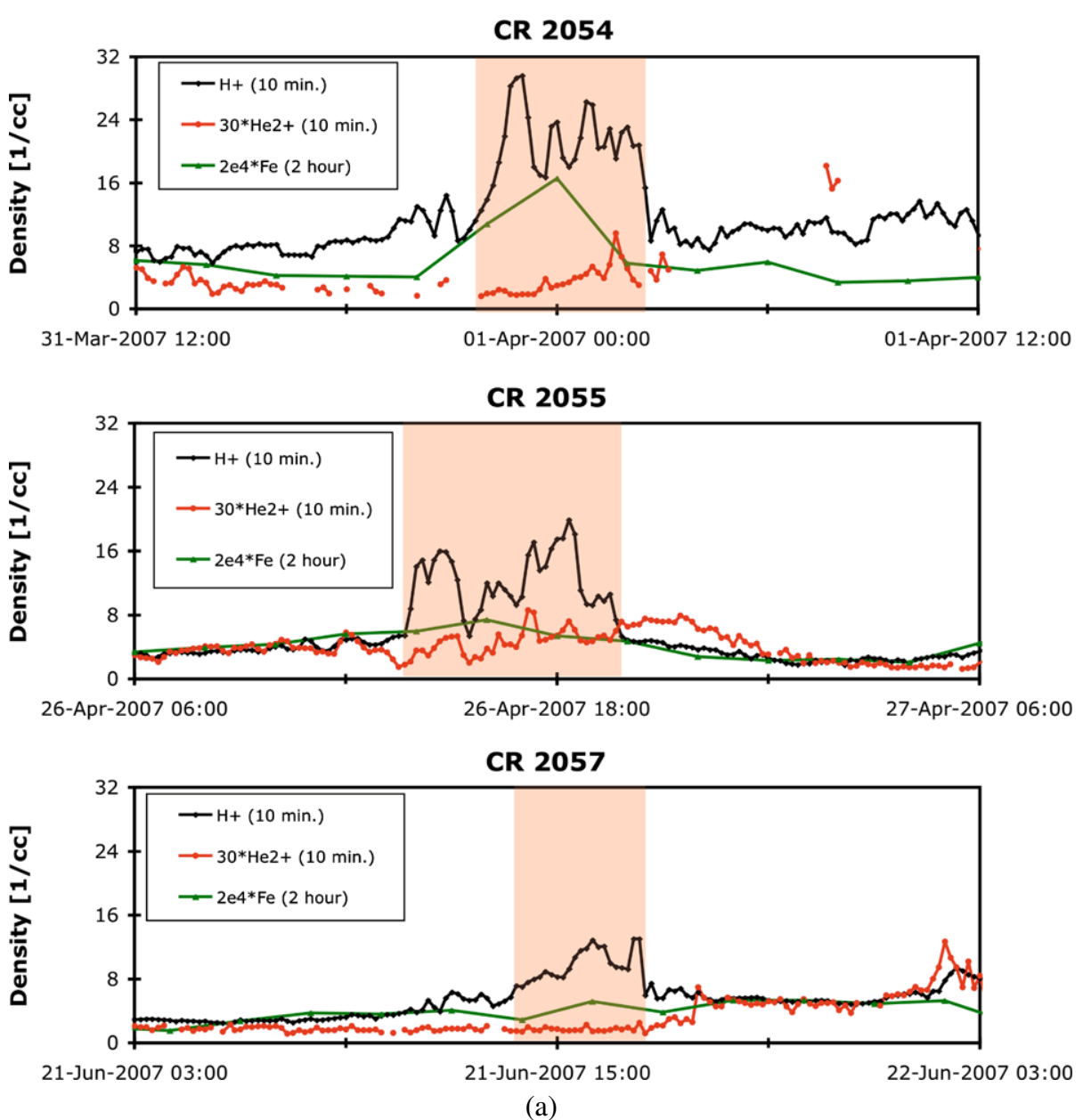

Figure 12 (a) Solar wind composition data from ST-A. 10 minute averaged proton density is shown in black, 10 minute alpha density (multiplied by a factor of 30) is shown in red, and 2 hour accumulated iron density (multiplied by a factor of $2 \times 10^{4}$ ) is shown in green. The shaded regions indicate the HPS. From top to bottom are CR 2054, 2055, and 2057. (b) Solar wind composition data from ST-B. 10 minute averaged proton density is shown in black, 10 minute alpha density (multiplied by a factor of 30) is shown in red, and 2 hour accumulated iron density (in arbitrary units) is shown in green. The shaded regions indicate the HPS. From top to bottom are CR 2054, 2055, and 2057. (c) Solar wind composition data from ACE. 1 hour averaged proton density is shown in black, 1 hour averaged alpha density (multiplied by a factor of 30) is shown in red, and 2 hour accumulated iron density (multiplied by a factor of $2 \times 10^{4}$ ) is shown in green. The shaded regions indicate the HPS. From top to bottom are CR 2054, 2055, and 2057.

is less than or equal to the increase in protons. Gravitational stratification would result in a greater depletion of iron than helium (Wimmer-Schweingruber, 2002), so our observations do not support gravitational stratification at the solar source region for the cause of He depletion relative to protons in the vicinity of the HPS. This is consistent with the results of Wimmer-Schweingruber (1994). Evidence for gravitational settling in the cores of streamers has been presented in studies such as Raymond et al. (1997) and Uzzo et al. (2003). The HPS material observed in this study is unlikely to have originated in streamer cores. 


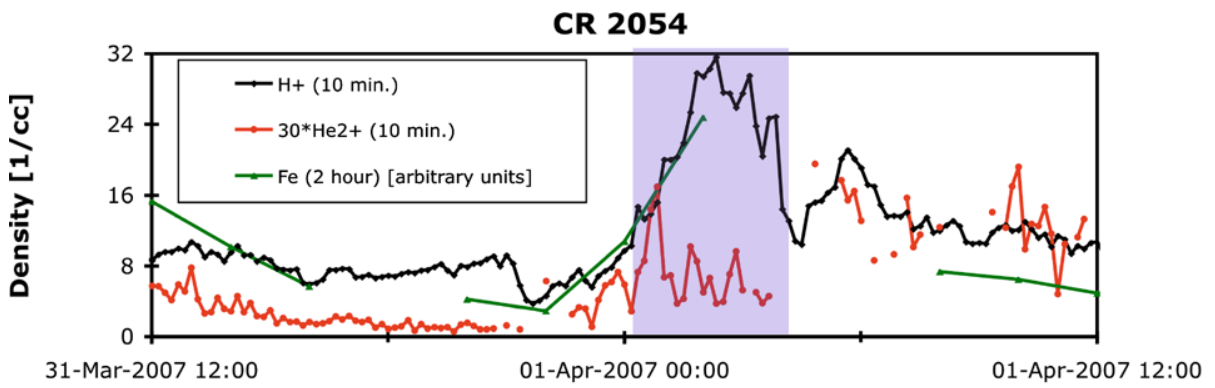

CR 2055

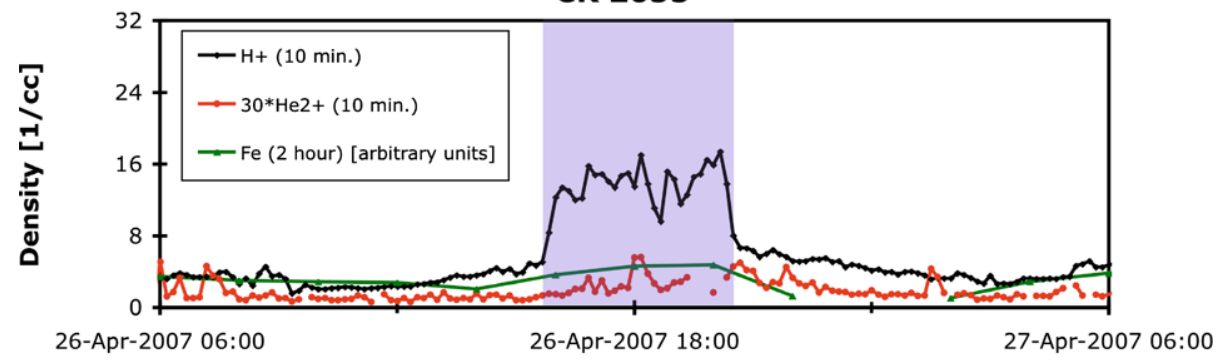

CR 2057

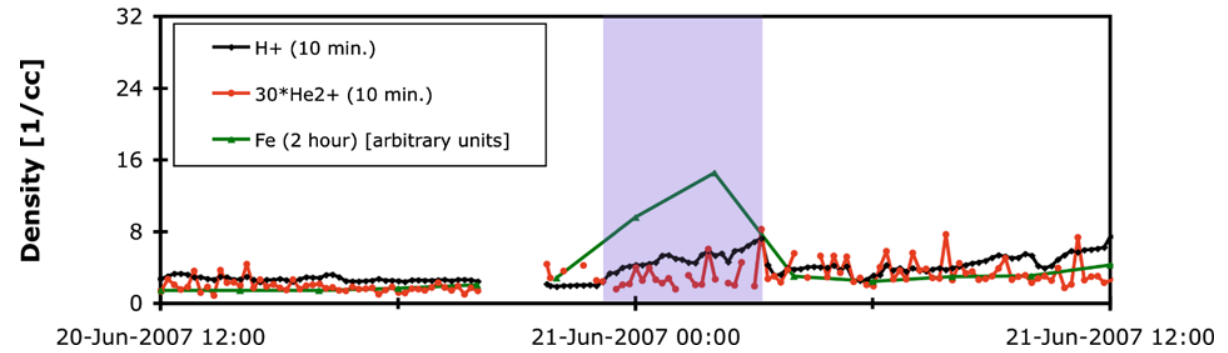

(b)

Figure 12 (Continued.)

To sum up: Based on three-point observations, the undisturbed HPS was found to lie roughly parallel to the idealized Parker spiral in the ecliptic plane. The location of the HCS within the plasma sheet was similar between all three spacecraft within a Carrington rotation, indicating that the HPS is quasi-stationary over the time scale of about a day, rather than transient in nature. However, the HPS clearly evolved from one solar rotation to the next. A further study is planned to narrow the range over which the HPS can be described as quasi-stationary. Next, the kinetic solar wind parameters associated with the HPS observed at the respective spacecraft were not identical, even though the temporal separation between observations was small, less than 1 day. Separation in heliographic latitude, though small, is the most likely explanation for the disparities between observation points. Lastly, in the absence of ICME material we observed a modest enhancement in the density of iron ions with each crossing of the HPS, similar to the observations of Schmid, Bochsler, and Geiss (1988). This supports the conclusion of Wimmer-Schweingruber (1994) that gravitational stratification at the solar source region is not responsible for the well-observed $N_{\mathrm{a}} / N_{\mathrm{p}}$ de- 

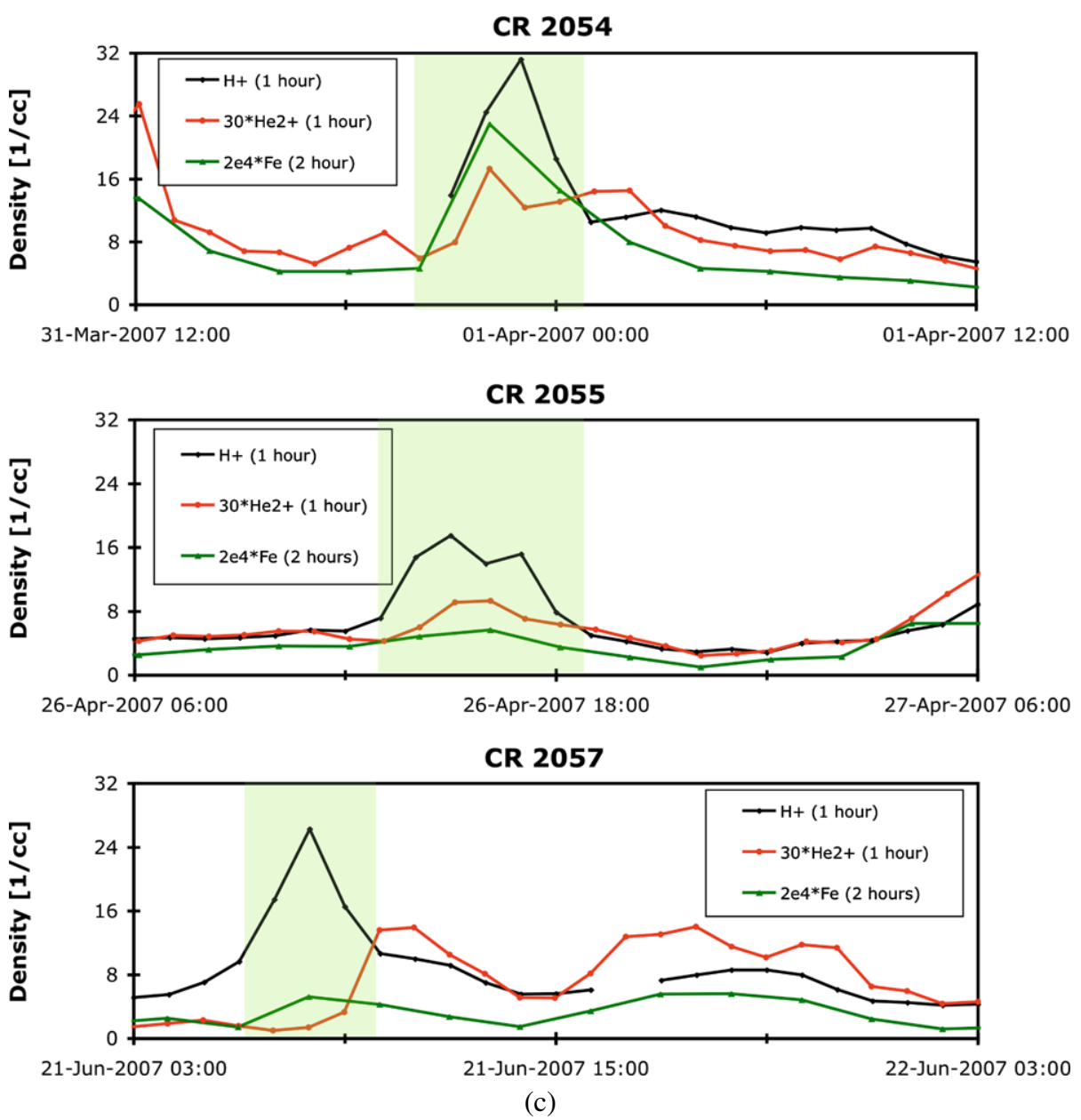

Figure 12 (Continued.)

pletion associated with encounters of the HPS. This suggests the source of the HPS material sampled in this study was not streamer cores.

Acknowledgements This work was supported at UNH under NASA contract NAS5-00132, and grant NNX10AQ29G.

K.D.C. Simunac would like to thank Claire Foullon and Robert Wimmer-Schweingruber for helpful discussions.

We thank the STEREO/SECCHI team for making their data available.

We thank the ACE SWEPAM and ACE SWICS instrument teams, and the ACE Science Center for providing the ACE data.

This work utilizes data obtained by the Global Oscillation Network Group (GONG) Program, managed by the National Solar Observatory, which is operated by AURA, Inc. under a cooperative agreement with the National Science Foundation. The data were acquired by instruments operated by the Big Bear Solar Observatory, High Altitude Observatory, Learmonth Solar Observatory, Udaipur Solar Observatory, Instituto de Astrofísica de Canarias, and Cerro Tololo Interamerican Observatory.

Simulation results have been provided by the Community Coordinated Modeling Center at Goddard Space Flight Center through their public Runs on Request system (http://ccmc.gsfc.nasa.gov). The CCMC is a 
multi-agency partnership between NASA, AFMC, AFOSR, AFRL, AFWA, NOAA, NSF and ONR. The Wang-Sheeley-Arge (WSA) Model was developed by Nick Arge at AFRL.

Open Access This article is distributed under the terms of the Creative Commons Attribution License which permits any use, distribution, and reproduction in any medium, provided the original author(s) and the source are credited.

\section{References}

Acuña, M.H., Ogilvie, K.W., Baker, D.N., Curtis, S.A., Fairfield, D.H., Mish, W.H.: 1995, Space Sci. Rev. 71, 5 .

Acuña, M.H., Curtis, D., Scheifele, J.L., Russell, C.T., Schroeder, P., Szabo, A., Luhmann, J.G.: 2008, Space Sci. Rev. 136, 203.

Bavassano, B., Woo, R., Bruno, R.: 1997, Geophys. Res. Lett. 24, 1655.

Borrini, G., Gosling, J.T., Bame, S.J., Feldman, W.C., Wilcox, J.M.: 1981, J. Geophys. Res. 86, 4565.

Burlaga, L.F.: 1974, J. Geophys. Res. 79, 3717.

Burlaga, L.F., Mish, W.H., Whang, Y.C.: 1990, J. Geophys. Res. 95, 4247.

Crooker, N.U., Gosling, J.T., Kahler, S.W.: 1998, J. Geophys. Res. 103, 301.

Crooker, N.U., Burton, M.E., Siscoe, G.L., Kahler, S.W., Gosling, J.T., Smith, E.J.: 1996, J. Geophys. Res. 101, 24,331.

Crooker, N.U., McAllister, A.H., Fitzenreiter, R.J., Linker, J.A., Larson, D.E., Lepping, R.P., Szabo, A., Steinberg, J.T., Lazarus, A.J., Mikic, Z., Lin, R.P.: 1998, J. Geophys. Res. 103, 26,859.

Crooker, N.U., Huang, C.-L., Lamassa, S.M., Larson, D.E., Kahler, S.W., Spence, H.E.: 2004, J. Geophys. Res. 109, A03107.

Foullon, C., Lavraud, B., Wardle, N.C., Owen, C.J., Kucharek, H., Fazakerley, A.N., Larson, D.E., Lucek, E., Luhmann, J.G., Opitz, A., Sauvaud, J.-A., Skoug, R.M.: 2009, Solar Phys. 259, 389.

Galvin, A.B., Kistler, L.M., Popecki, M.A., Farrugia, C.J., Simunac, K.D.C., Ellis, L., Möbius, E., Lee, M.A., Boehm, M., Carroll, J., Crawshaw, A., Conti, M., Demaine, P., Ellis, S., Gaidos, J.A., Googins, J., Granoff, M., Gustafson, A., Heirtzler, D., King, B., Knauss, U., Levasseur, J., Longworth, S., Singer, K., Turco, S., Vachon, P., Vosbury, M., Widholm, M., Blush, L.M., Karrer, R., Bochsler, P., Daoudi, H., Etter, A., Fischer, J., Jost, J., Opitz, A., Sigrist, M., Wurz, P., Klecker, B., Ertl, M., Seidenschwang, E., Wimmer-Schweingruber, R.F., Koeten, M., Thompson, B., Steinfeld, D.: 2008, Space Sci. Rev. 136, 437.

Geiss, J., Gloeckler, G., von Steiger, R.: 1995, Space Sci. Rev. 72, 49.

Gloeckler, G., Cain, J., Ipavich, F.M., Turns, E.O., Bedini, P., Fisk, L.A., Zurbuchen, T.H., Bochsler, P., Fischer, J., Wimmer-Schweingruber, R.F., Geiss, J., Kallenbach, R.: 1998, Space Sci. Rev. 86, 497.

Gosling, J.T., Asbridge, J.R., Bame, S.J., Feldman, W.C.: 1978, J. Geophys. Res. 83, 1401.

Gosling, J.T., Borrini, G., Asbridge, J.R., Bame, S.J., Feldman, W.C., Hansen, R.T.: 1981, J. Geophys. Res. 86, 5438 .

Howard, R.A., Moses, J.D., Vourlidas, A., Newmark, J.S., Socker, D.G., Plunkett, S.P., Korendyke, C.M., Cook, J.W., Hurley, A., Davila, J.M., Thompson, W.T., St. Cyr, O.C., Mentzell, E., Mehalick, K., Lemen, J.R., Wuelser, J.P., Duncan, D.W., Tarbell, T.D., Wolfson, C.J., Moore, A., Harrison, R.A., Waltham, N.R., Lang, J., Davis, C.J., Eyles, C.J., Mapson-Menard, H., Simnett, G.M., Halain, J.P., Defise, J.M., Mazy, E., Rochus, P., Mercier, R., Ravet, M.F., Delmotte, F., Auchere, F., Delaboudiniere, J.P., Bothmer, V., Deutsch, W., Wang, D., Rich, N., Cooper, S., Stephens, V., Maahs, G., Baugh, R., McMullin, D., Carter, T. (eds.): 2008, Space Sci. Rev. 136, 67.

Kaiser, M.L., Kucera, T.A., Davila, J.M., St. Cyr, O.C., Guhathakurta, M., Christian, E.: 2008, Space Sci. Rev. 136, 5.

Kilpua, E.K.J., Liewer, P.C., Farrugia, C., Luhmann, J.G., Möstl, C., Li, Y., Liu, Y., Lynch, B.J., Russell, C.T., Vourlidas, A., Acuna, M.H., Galvin, A.B., Larson, D., Sauvaud, J.A.: 2009, Solar Phys. 254, 325.

Lepping, R.P., Acuña, M.H., Burlaga, L.F., Farrell, W.M., Slavin, J.A., Schatten, K.H., Mariani, F., Ness, N.F., Neubauer, F.M., Whang, Y.C., Byrnes, J.B., Kennon, R.S., Panetta, P.V., Scheifele, J., Worley, E.M.: 1995, Space Sci. Rev. 71, 207.

Liu, Y.C.-M., Galvin, A.B., Popecki, M.A., Simunac, K.D.C., Kistler, L., Farrugia, C., Lee, M.A., Klecker, B., Bochsler, P., Luhmann, J.L., Jian, L.K., Moebius, E., Wimmer-Schweingruber, R., Wurz, P.: 2010a, In: Maksimovic, M., Issautier, K., Meyer-Vernet, N., Moncuquet, M., Pantellini, F. (eds.) Twelfth International Solar Wind Conference, Melville, New York, AIP CS-1216, 363.

Liu, Y., Galvin, A.B., Popecki, M., Simunac, K., Kistler, L.M., Farrugia, C.J., Moebius, E., Jian, L., Opitz, A., Luhmann, J.G.: 2010b, American Geophysical Union Fall Meeting, abstract \#SH33C-05. 
Luhmann, J.G., Curtis, D.W., Schroeder, P., McCauley, J., Lin, R.P., Larson, D.E., Bale, S.D., Sauvaud, J.-A., Aoustin, C., Mewaldt, R.A., Cummings, A.C., Stone, E.C., Davis, A.J., Cook, W.R., Kecman, B., Wiedenbeck, M.E., von Rosenvinge, T., Acuna, M.H., Reichenthal, L.S., Shuman, S., Wortman, K.A., Reames, D.V., Mueller-Mellin, R., Kunow, H., Mason, G.M., Walpole, P., Korth, A., Sanderson, T.R., Russell, C.T., Gosling, J.T.: 2008, Space Sci. Rev. 136, 117.

McComas, D.J., Bame, S.J., Barker, P., Feldman, W.C., Phillips, J.L., Riley, P., Griffee, J.W.: 1998, Space Sci. Rev. 86, 563.

Möstl, C., Farrugia, C.J., Biernat, H.K., Leitner, M., Kilpua, E.K.J., Galvin, A.B., Luhmann, J.G.: 2009, Solar Phys. 256, 427.

Newton, H.W., Nunn, M.L.: 1951, Mon. Not. Roy. Astron. Soc. 111, 413.

Ogilvie, K.W., Chornay, D.J., Fritzenreiter, R.J., Hunsaker, F., Keller, J., Lobell, J., Miller, G., Scudder, J.D., Sittler, E.C. Jr., Torbert, R.B., Bodet, D., Needell, G., Lazarus, A.J., Steinberg, J.T., Tappan, J.H., Mavretic, A., Gergin, E.: 1995, Space Sci. Rev. 71, 55.

Opitz, A.: 2007, Ph.D. Dissertation, University of Bern.

Raymond, J.C., Kohl, J.L., Noci, G., Antonucci, E., Tondello, G., Huber, M.C.E., Gardner, L.D., Nicolosi, P., Fineschi, S., Romoli, M., Spadaro, D., Siegmund, O.H.W., Benna, C., Ciaravella, A., Cranmer, S., Giordano, S., Krovska, M., Martin, R., Michels, J., Modigliani, A., Naletto, G., Panaskuk, A., Pernechele, C., Poletto, G., Smith, P.L., Suleiman, R.M., Strachan, L.: 1997, Solar Phys. 175, 645.

Sauvaud, J.-A., Larson, D., Aoustin, C., Curtis, D., Médale, J.-L., Fedorov, A., Rouzaud, J., Luhmann, J., Moreau, T., Schroeder, P., Louarn, P., Dandouras, I., Penou, E.: 2008, Space Sci. Rev. 136, 227.

Schmid, J., Bochsler, P., Geiss, J.: 1988, Astrophys. J. 329, 956.

Schwenn, R.: 1990, In: Schwenn, R., Marsch, E. (eds.) Physics of the Inner Heliosphere, Springer, Berlin, 99.

Simunac, K.D.C.: 2009, Ph.D. Dissertation, University of New Hampshire.

Siscoe, G., Intriligator, D.: 1993, Geophys. Res. Lett. 20, 2267.

Stone, E.C., Frandsen, A.M., Mewaldt, R.A., Christian, E.R., Margolies, D., Ormes, J.F., Snow, F.: 1998, Space Sci. Rev. 86, 1.

Suess, S., Ko, Y.-K., von Steiger, R., Moore, R.L.: 2009, J. Geophys. Res. 114, A04103.

Uzzo, M., Ko, Y.-K., Raymond, J.C., Wurz, P., Ipavich, F.M.: 2003, Astrophys. J. 585, 1062.

Wang, Y.-M., Sheeley, N.R., Rich, N.B.: 2007, Astrophys. J. 658, 1340.

Wimmer-Schweingruber, R.F.: 1994, Ph.D. Dissertation, University of Bern.

Wimmer-Schweingruber, R.F.: 2002, Adv. Space Res. 30, 23.

Winterhalter, D., Smith, E.J., Burton, M.E., Murphy, N., McComas, D.J.: 1994, J. Geophys. Res. $99,6667$.

Wilcox, J.M., Ness, N.F.: 1965, J. Geophys. Res. 70, 5793. 